\title{
Well-posedness and Stability of the Repairable System with Three Units and Vacation
}

\author{
Xiaoshuang HAN \\ College of Science \& Technology, Yanbian University, Yanji 133002, China \\ E-mail: yustxy@hotmail.com \\ Mingyan TENG \\ Department of Mathematics, Bohai University, Jinzhou 121002, China \\ E-mail: fz555888@aliyun.com \\ Ming FANG ${ }^{\dagger}$ \\ Department of Mathematics, Science College, Yanbian University, Yanji 133002, China \\ E-mail: fangming@ybu.edu.cn
}

\begin{abstract}
The stability of the repairable system with three units and vacation was investigated by two different methods in this note. The repairable system is described by a set of ordinary differential equation coupled with partial differential equations with initial values and integral boundaries. To apply the theory of positive operator semigroups to discuss the repairable system, the system equations were transformed into an abstract Cauchy problem on some Banach lattice. The system equations have a unique non-negative dynamic solution and positive steady-state solution and dynamic solution strongly converges to steady-state solution were shown on the basis of the detailed spectral analysis of the system operator. Furthermore, the Cesàro mean ergodicity of the semigroup $T(t)$ generated by the system operator was also shown through the irreducibility of the semigroup.
\end{abstract}

Keywords repairable system; positive operator semigroups; spectral analysis; stability

\section{Introduction}

Repairable system is not only an important class of systems discussed in reliability theory but also one of main objects studied in reliability mathematics. It consists of some components under the supervision of one or more repairmen. If a component fails at any time, it is immediately sent to the repair facility for repair.

The repairable system with three units is frequently encountered in practice. Many authors have discussed this repairable system where the repairmen always remain idle until the failed units present. For instance, Song and Deng analyzed the reliability of a three-unit system in a changing environment in [1]. Li et al. studied a repairable system with three units and two different repair facilities, and derived the explicit expressions of the state probabilities of the system and the steady-state reliability characteristics of the system in [2]. Kovalenko

Received November 11, 2013, accepted January 3, 2014

†Corresponding author: E-mail: fangming@ybu.edu.cn 
investigated a three-components system consisting of one master control element and two slave elements with priority serving by a single repair facility, and obtained the readiness factor and the average up-time in [3]. $\mathrm{Hu}$ et al. discussed a three-unit system with $n$ failure modes and priority, and obtained explicit expressions of the steady-state probabilities of the system in [4].

The vacation conceptions (single vacation, multiple vacation, and delay vacation ${ }^{[5]}$ ) were first introduced into the repairable system by Su and Shi in [6]. The three-unit repairable system with vacations were discussed by $\mathrm{Hu}$ and his collaborators in recent years. $\mathrm{Hu}$ et al discussed the three-unit system with multiple vacations and priority in [7] and [8]. The three-unit system with single vacations was established and its reliability was then studied in [9]. They defined the repairable system and derived integro-differential equations, which describe the system precisely. By transforming the system equations into the first-order ordinary differential equations, they obtained the explicit formulas for the steady-state solution, the steady-state availability and mean up-time of the system.

However, the above authors investigated the repairable systems from the reliability theory only and mainly devoted to obtaining the quantities related to reliability of the system. They focused not at all on the problems of uniqueness, existence and asymptotic stability of the dynamic solutions to the system equations. We shall concern these questions of the repairable system with three units and single vacation in this paper. Indeed, the well-posedness and stability of the repairable systems have attracted the attention of many researchers since the end of last century. The corresponding properties of various repairable systems without vacation were obtained by the $C_{0}$ semigroups theory of functional analysis in [10-19].

Though several different methods given by the authors of [10-19] were given to deal with the well-posedness and stability of the repairable systems, it is not easy to obtain the wellposedness and stability of the repairable system with vacation due to the complexity of the system equations. Analyzing the methods of [10-19] in length, we find out that the spectral analysis of the system operator plays important role in those methods. So we begin this paper with the spectral analysis. On the basis of the detailed spectral analysis of the system operator, we show that the $C_{0}$ semigroup $T(t)$ generated by the system operator is positive, contractive and irreducible. Thus the well-posedness of the system is obtained, that is the system equations have a unique non-negative dynamic solution. Moreover, we show the system equations have positive steady-state solution and dynamic solution strongly converges to steady-state solution by two different methods. One way follows from the extension theorem of Lyubich and Phong in [20]. Another way is based on the irreducibility of the semigroups.

The structure of this paper is organized as follows. In section 2, we will introduce the repairable system with three units and vacation. The state space and system operator are also introduced in this section. In section 3 , several lemmas and corollaries are given to obtain the spectral distributions of the system operator. In section 4, we analyze the spectral distribution of the system operator. In the section 5, main results of this paper are obtained.

\section{System equations and system operator}

Following [9], the system considered here consists of three dissimilar units (called units 1, 2 and 3) and one repairman with single vacation. Initially, the system with three new units 
begins to operate. The repairman does not take a vacation and remain idle until the first failed unit appears. A repaired unit is as good as a new one. The failure time distributions are exponential, and the repair time distributions are general: $\lambda_{k}$ and $\mu_{k}(x)$ are the failure rates and repair rate functions of unit $k(k=1,2,3)$. The vacation time distribution is assumed to be general as well, $\eta(x)$ is the vacation rate function. By utilizing the similar method of [9], we are able to obtain the integro-differential equations

$$
\begin{aligned}
& {\left[\frac{\mathrm{d}}{\mathrm{d} t}+\lambda_{1}+\lambda_{2}+\lambda_{3}\right] p_{0}(t)=\int_{0}^{\infty} \eta(u) q_{7}(u, t) \mathrm{d} u} \\
& {\left[\frac{\partial}{\partial t}+\frac{\partial}{\partial x}+\mu_{1}(x)\right] q_{1}(x, t)=0} \\
& {\left[\frac{\partial}{\partial t}+\frac{\partial}{\partial y}+\lambda_{1}+\lambda_{3}+\mu_{2}(y)\right] q_{2}(y, t)=\int_{0}^{\infty} \mu_{1}(x) q_{4}(x, y, t) \mathrm{d} x} \\
& {\left[\frac{\partial}{\partial t}+\frac{\partial}{\partial z}+\lambda_{1}+\lambda_{2}+\mu_{3}(z)\right] q_{3}(z, t)=\int_{0}^{\infty} \mu_{1}(x) q_{5}(x, z, t) \mathrm{d} x+\int_{0}^{\infty} \mu_{2}(y) q_{6}(y, z, t) \mathrm{d} y(4)} \\
& {\left[\frac{\partial}{\partial t}+\frac{\partial}{\partial x}+\mu_{1}(x)\right] q_{4}(x, y, t)=0} \\
& {\left[\frac{\partial}{\partial t}+\frac{\partial}{\partial x}+\mu_{1}(x)\right] q_{5}(x, z, t)=0} \\
& {\left[\frac{\partial}{\partial t}+\frac{\partial}{\partial y}+\mu_{2}(y)\right] q_{6}(y, z, t)=0} \\
& {\left[\frac{\partial}{\partial t}+\frac{\partial}{\partial u}+\lambda_{1}+\lambda_{2}+\lambda_{3}+\eta(u)\right] q_{7}(u, t)=0} \\
& {\left[\frac{\partial}{\partial t}+\frac{\partial}{\partial u}+\eta(u)\right] q_{8}(u, t)=\lambda_{1} q_{7}(u, t)} \\
& {\left[\frac{\partial}{\partial t}+\frac{\partial}{\partial u}+\lambda_{1}+\lambda_{3}+\eta(u)\right] q_{9}(u, t)=\lambda_{2} q_{7}(u, t)} \\
& {\left[\frac{\partial}{\partial t}+\frac{\partial}{\partial u}+\lambda_{1}+\lambda_{2}+\eta(u)\right] q_{10}(u, t)=\lambda_{3} q_{7}(u, t)} \\
& {\left[\frac{\partial}{\partial t}+\frac{\partial}{\partial u}+\eta(u)\right] q_{11}(u, t)=\lambda_{1} q_{9}(u, t)} \\
& {\left[\frac{\partial}{\partial t}+\frac{\partial}{\partial u}+\eta(u)\right] q_{12}(u, t)=\lambda_{1} q_{10}(u, t)} \\
& {\left[\frac{\partial}{\partial t}+\frac{\partial}{\partial u}+\eta(u)\right] q_{13}(u, t)=\lambda_{2} q_{10}(u, t)+\lambda_{3} q_{9}(u, t)} \\
& {\left[\frac{\partial}{\partial t}+\frac{\partial}{\partial x}+\mu_{1}(x)\right] q_{14}(x, t)=0} \\
& {\left[\frac{\partial}{\partial t}+\frac{\partial}{\partial x}+\mu_{1}(x)\right] q_{15}(x, t)=0} \\
& {\left[\frac{\partial}{\partial t}+\frac{\partial}{\partial y}+\mu_{2}(y)\right] q_{16}(y, t)=\lambda_{3} q_{2}(y, t)}
\end{aligned}
$$

with boundary conditions:

$$
q_{1}(0, t)=\lambda_{1} p_{0}(t)+\int_{0}^{\infty} \eta(u) q_{8}(u, t) \mathrm{d} u
$$




$$
\begin{aligned}
& q_{2}(0, t)=\lambda_{2} p_{0}(t)+\int_{0}^{\infty} \eta(u) q_{9}(u, t) \mathrm{d} u+\int_{0}^{\infty} \mu_{1}(x) q_{14}(x, t) \mathrm{d} x \\
& q_{3}(0, t)=\lambda_{3} p_{0}(t)+\int_{0}^{\infty} \eta(u) q_{10}(u, t) \mathrm{d} u+\int_{0}^{\infty} \mu_{1}(x) q_{15}(x, t) \mathrm{d} x+\int_{0}^{\infty} \mu_{2}(y) q_{16}(y, t) \mathrm{d} y(20) \\
& q_{4}(0, y, t)=\lambda_{1} q_{2}(y, t), \quad q_{5}(0, z, t)=\lambda_{1} q_{3}(z, t), \quad q_{6}(0, z, t)=\lambda_{2} q_{3}(z, t) \\
& q_{7}(0, t)=\int_{0}^{\infty} \mu_{1}(x) q_{1}(x, t) \mathrm{d} x+\int_{0}^{\infty} \mu_{2}(y) q_{2}(y, t) \mathrm{d} y+\int_{0}^{\infty} \mu_{3}(z) q_{3}(z, t) \mathrm{d} z \\
& q_{i}(0, t)=0, \quad i=8, \cdots, 13 \\
& q_{j}(0, t)=\int_{0}^{\infty} \eta(u) q_{j-3}(u, t) \mathrm{d} u, \quad j=14,15,16
\end{aligned}
$$

and the only nonzero initial condition is $p_{0}(0)=1$.

The state space is chosen as

$$
X=\mathbb{R} \times\left(L^{1}\left(\mathbb{R}^{+}\right)\right)^{3} \times\left(L^{1}\left(\mathbb{R}^{+} \times \mathbb{R}^{+}\right)\right)^{3} \times\left(L^{1}\left(\mathbb{R}^{+}\right)\right)^{10} .
$$

Obviously, $X$ is a Banach space with the norm

$$
\|\vec{P}\|=\left|p_{0}\right|+\sum_{i=4}^{6} \int_{\mathbb{R}^{+} \times \mathbb{R}^{+}}\left|p_{i}(x, y)\right| \mathrm{d} x \mathrm{~d} y+\sum_{i=1, i \neq 4,5,6}^{16} \int_{\mathbb{R}^{+}}\left|p_{i}(x)\right| \mathrm{d} x,
$$

in which

$$
\vec{P}=\left(p_{0}, p_{1}(x), p_{2}(x), p_{3}(x), p_{4}(x, y), p_{5}(x, y), p_{6}(x, y), p_{7}(x), \cdots, p_{16}(x)\right) \in X
$$

To introduce the system operator, we need the identities

$$
\begin{aligned}
& p_{1}(0)=\lambda_{1} p_{0}+\int_{0}^{\infty} \eta(x) p_{8}(x) \mathrm{d} x \\
& p_{2}(0)=\lambda_{2} p_{0}+\int_{0}^{\infty} \eta(x) p_{9}(x) \mathrm{d} x+\int_{0}^{\infty} \mu_{1}(x) p_{14}(x) \mathrm{d} x \\
& p_{3}(0)=\lambda_{3} p_{0}+\int_{0}^{\infty} \eta(x) p_{10}(x) \mathrm{d} x+\int_{0}^{\infty} \mu_{1}(x) p_{15}(x) \mathrm{d} x+\int_{0}^{\infty} \mu_{2}(x) p_{16}(x) \mathrm{d} x \\
& p_{4}(0, y)=\lambda_{1} p_{2}(y), p_{5}(0, y)=\lambda_{1} p_{3}(y), p_{6}(0, y)=\lambda_{2} p_{3}(y) \\
& p_{7}(0)=\int_{0}^{\infty} \mu_{1}(x) p_{1}(x) \mathrm{d} x+\int_{0}^{\infty} \mu_{2}(x) p_{2}(x) \mathrm{d} x+\int_{0}^{\infty} \mu_{3}(x) p_{3}(x) \mathrm{d} x \\
& p_{i}(0)=0, i=8, \cdots, 13 \quad j=14,15,16 \\
& p_{j}(0)=\int_{0}^{\infty} \eta(x) p_{j-3}(x) \mathrm{d} x, \quad j=16
\end{aligned}
$$

Thus, from the system equations of $(1) \sim(24)$, the system operator $A$ defined in the state space $X$ is given by

$$
D(A)=\left\{\begin{array}{l|l}
\vec{P} \in X & \begin{array}{l}
p_{i}(x) \text { and } p_{j}(x, y) \text { are absolutely continuous, } p_{i}^{\prime}(x) \in \\
L^{1}\left(\mathbb{R}^{+}\right), \frac{\partial}{\partial x} p_{j}(x, y) \in L^{1}\left(\mathbb{R}^{+} \times \mathbb{R}^{+}\right),(j=4,5,6 ; i=1, \\
2,3,7, \cdots, 16) \text { and they satisfy the identities }(25) \sim(31) .
\end{array}
\end{array}\right\}
$$




$$
A \vec{P}=\left(\begin{array}{l}
-\left[\lambda_{1}+\lambda_{2}+\lambda_{3}\right] p_{0}+\int_{0}^{\infty} \eta(x) p_{7}(x) \mathrm{d} x \\
-\left[\frac{\mathrm{d}}{\mathrm{d} x}+\mu_{1}(x)\right] p_{1}(x) \\
-\left[\frac{\mathrm{d}}{\mathrm{d} y}+\lambda_{1}+\lambda_{3}+\mu_{2}(y)\right] p_{2}(y)+\int_{0}^{\infty} \mu_{1}(x) p_{4}(x, y) \mathrm{d} x \\
-\left[\frac{\mathrm{d}}{\mathrm{d} y}+\lambda_{1}+\lambda_{2}+\mu_{3}(y)\right] p_{3}(y)+\int_{0}^{\infty} \mu_{1}(x) p_{5}(x, y) \mathrm{d} x+ \\
\quad \int_{0}^{\infty} \mu_{2}(x) p_{6}(x, y) \mathrm{d} x \\
-\left[\frac{\partial}{\partial x}+\mu_{1}(x)\right] p_{4}(x, y) \\
-\left[\frac{\partial}{\partial x}+\mu_{1}(x)\right] p_{5}(x, y) \\
-\left[\frac{\partial}{\partial x}+\mu_{2}(x)\right] p_{6}(x, y) \\
-\left[\frac{\mathrm{d}}{\mathrm{d} x}+\lambda_{1}+\lambda_{2}+\lambda_{3}+\eta(x)\right] p_{7}(x) \\
-\left[\frac{\mathrm{d}}{\mathrm{d} x}+\eta(x)\right] p_{8}(x)+\lambda_{1} p_{7}(x) \\
-\left[\frac{\mathrm{d}}{\mathrm{d} x}+\lambda_{1}+\lambda_{3}+\eta(x)\right] p_{9}(x)+\lambda_{2} p_{7}(x) \\
-\left[\frac{\mathrm{d}}{\mathrm{d} x}+\lambda_{1}+\lambda_{2}+\eta(x)\right] p_{10}(x)+\lambda_{3} p_{7}(x) \\
-\left[\frac{\mathrm{d}}{\mathrm{d} x}+\eta(x)\right] p_{11}(x)+\lambda_{1} p_{9}(x) \\
-\left[\frac{\mathrm{d}}{\mathrm{d} x}+\eta(x)\right] p_{12}(x)+\lambda_{1} p_{10}(x) \\
-\left[\frac{\mathrm{d}}{\mathrm{d} x}+\eta(x)\right] p_{13}(x)+\lambda_{2} p_{10}(x)+\lambda_{3} p_{9}(x) \\
-\left[\frac{\mathrm{d}}{\mathrm{d} x}+\mu_{1}(x)\right] p_{14}(x) \\
-\left[\frac{\mathrm{d}}{\mathrm{d} x}+\mu_{1}(x)\right] p_{15}(x) \\
-\left[\frac{\mathrm{d}}{\mathrm{d} x}+\mu_{2}(x)\right] p_{16}(x)+\lambda_{3} p_{2}(x)
\end{array}\right)
$$

From the above definitions of the state space and the operator $A$, the equations $(1) \sim(24)$ is equivalent to the following abstract Cauchy problem on the Banach space $X$.

$$
\left\{\begin{array}{l}
\frac{\mathrm{d}}{\mathrm{d} t} \vec{P}(t)=A \vec{P}(t), \quad \forall t \geq 0, \vec{P}(t) \in X \\
\vec{P}(0)=(1,0, \cdots, 0)^{\mathrm{T}}
\end{array}\right.
$$

\section{Lemmas and corollaries}

The main results are obtained under the following hypotheses.

General Hypotheses $\mu_{i}(x)(i=1,2,3)$ and $\eta(x)$ are bounded functions satisfying $\int_{0}^{\infty} \mu_{j}(x) \mathrm{d} x=\infty$ and $\int_{0}^{\infty} \eta(x) \mathrm{d} x=\infty$. Moreover, there exist positive constants $M_{j}$ and $c_{j}$ $(j=1,2,3,4)$ such that $0 \leq \mu_{i}(x) \leq M_{i}, 0 \leq \eta(x) \leq M_{4}$ and $\forall x \in \mathbb{R}^{+}, \frac{1}{x} \int_{0}^{x} \mu_{i}(x) \mathrm{d} x>c_{i}$, $\frac{1}{x} \int_{0}^{x} \eta(x) \mathrm{d} x>c_{4}$.

It is easy to obtain the following lemmas and corollaries by the general hypotheses. The proofs of these lemmas and corollaries are given in the appendix of this note.

Lemma 1 For the repair rate functions $\mu_{i}(x)(i=1,2,3)$ and vacation rate function $\eta(x)$, we have

$$
\int_{0}^{\infty} \mu_{i}(x) \mathrm{e}^{-\int_{0}^{x} \mu_{i}(s) \mathrm{d} s} \mathrm{~d} x=1, \quad \int_{0}^{\infty} \eta(x) \mathrm{e}^{-\int_{0}^{x} \eta(s) \mathrm{d} s} \mathrm{~d} x=1 .
$$

Lemma 2 If let

$$
a_{1}=\int_{0}^{\infty} \eta(x) \mathrm{e}^{-\int_{0}^{x} \lambda_{1}+\lambda_{2}+\lambda_{3}+\eta(s) \mathrm{d} s} \mathrm{~d} x,
$$




$$
\begin{aligned}
& a_{2}=\lambda_{1} \int_{0}^{\infty} \eta(x) \mathrm{e}^{-\int_{0}^{x} \eta(s) \mathrm{d} s} \int_{0}^{x} \mathrm{e}^{-\left(\lambda_{1}+\lambda_{2}+\lambda_{3}\right) s} \mathrm{~d} s \mathrm{~d} x, \\
& a_{3}=\lambda_{2} \int_{0}^{\infty} \eta(x) \mathrm{e}^{-\int_{0}^{x} \lambda_{1}+\lambda_{3}+\eta(s) \mathrm{d} s} \int_{0}^{x} \mathrm{e}^{-\lambda_{2} s} \mathrm{~d} s \mathrm{~d} x, \\
& a_{4}=\lambda_{3} \int_{0}^{\infty} \eta(x) \mathrm{e}^{-\int_{0}^{x} \lambda_{1}+\lambda_{2}+\eta(s) \mathrm{d} s} \int_{0}^{x} \mathrm{e}^{-\lambda_{3} s} \mathrm{~d} s \mathrm{~d} x, \\
& a_{5}=\lambda_{1} \int_{0}^{\infty} \eta(x) \mathrm{e}^{-\int_{0}^{x} \eta(s) \mathrm{d} s} \int_{0}^{x}\left(\mathrm{e}^{-\left(\lambda_{1}+\lambda_{3}\right) s}-\mathrm{e}^{-\left(\lambda_{1}+\lambda_{2}+\lambda_{3}\right) s}\right) \mathrm{d} s \mathrm{~d} x, \\
& a_{6}=\lambda_{1} \int_{0}^{\infty} \eta(x) \mathrm{e}^{-\int_{0}^{x} \eta(s) \mathrm{d} s} \int_{0}^{x}\left(\mathrm{e}^{-\left(\lambda_{1}+\lambda_{2}\right) s}-\mathrm{e}^{-\left(\lambda_{1}+\lambda_{2}+\lambda_{3}\right) s}\right) \mathrm{d} s \mathrm{~d} x, \\
& a_{7}=\lambda_{3} \int_{0}^{\infty} \eta(x) \mathrm{e}^{-\int_{0}^{x} \eta(s) \mathrm{d} s} \int_{0}^{x}\left(\mathrm{e}^{-\left(\lambda_{1}+\lambda_{3}\right) s}-\mathrm{e}^{-\left(\lambda_{1}+\lambda_{2}+\lambda_{3}\right) s}\right) \mathrm{d} s \mathrm{~d} x, \\
& a_{8}=\lambda_{2} \int_{0}^{\infty} \eta(x) \mathrm{e}^{-\int_{0}^{x} \eta(s) \mathrm{d} s} \int_{0}^{x}\left(\mathrm{e}^{-\left(\lambda_{1}+\lambda_{2}\right) s}-\mathrm{e}^{-\left(\lambda_{1}+\lambda_{2}+\lambda_{3}\right) s}\right) \mathrm{d} s \mathrm{~d} x, \\
& b_{1}=\lambda_{3} \int_{0}^{\infty} \mu_{2}(x) \mathrm{e}^{-\int_{0}^{x} \mu_{2}(s) \mathrm{d} s} \int_{0}^{x} \mathrm{e}^{-\lambda_{3} s} \mathrm{~d} s \mathrm{~d} x, \\
& b_{2}=\int_{0}^{\infty} \mu_{2}(x) \mathrm{e}^{-\int_{0}^{x} \lambda_{3}+\mu_{2}(s) \mathrm{d} s} \mathrm{~d} x,
\end{aligned}
$$

then $\sum_{i=1}^{8} a_{i}=1$ and $b_{1}+b_{2}=1$.

Lemma 3 If $z \in \mathbb{C}$, Re $z>0$ or $z=a i, a \neq 0$, then the following inequalities hold

$$
\left|\int_{0}^{\infty} \eta(x) \mathrm{e}^{-\int_{0}^{x} z+\eta(s) \mathrm{d} s} \mathrm{~d} x\right|<1, \quad\left|\int_{0}^{\infty} \mu_{i}(x) \mathrm{e}^{-\int_{0}^{x} z+\mu_{i}(s) \mathrm{d} s} \mathrm{~d} x\right|<1 .
$$

We are able to derive the following corollaries from the above lemmas.

Corollary 1 If $z$ is the same as above, set

$$
\begin{aligned}
g_{0}= & \int_{0}^{\infty} \eta(x) \mathrm{e}^{-\int_{0}^{x} z+\lambda_{1}+\lambda_{2}+\lambda_{3}+\eta(s) \mathrm{d} s} \mathrm{~d} x \\
g_{1}= & \lambda_{1} \int_{0}^{\infty} \eta(x) \mathrm{e}^{-\int_{0}^{x} z+\eta(s) \mathrm{d} s} \int_{0}^{x} \mathrm{e}^{-\left(\lambda_{1}+\lambda_{2}+\lambda_{3}\right) s} \mathrm{~d} s \mathrm{~d} x, \\
g_{2}= & \int_{0}^{\infty} \eta(x)\left(\mathrm{e}^{-\int_{0}^{x} z+\lambda_{1}+\lambda_{3}+\eta(s) \mathrm{d} s}-\mathrm{e}^{-\int_{0}^{x} z+\lambda_{1}+\lambda_{2}+\lambda_{3}+\eta(s) \mathrm{d} s}\right) \mathrm{d} x, \\
g_{3}= & \int_{0}^{\infty} \eta(x)\left(\mathrm{e}^{-\int_{0}^{x} z+\lambda_{1}+\lambda_{2}+\eta(s) \mathrm{d} s}-\mathrm{e}^{-\int_{0}^{x} z+\lambda_{1}+\lambda_{2}+\lambda_{3}+\eta(s) \mathrm{d} s}\right) \mathrm{d} x, \\
g_{4}= & \lambda_{1} \int_{0}^{\infty} \eta(x) \mathrm{e}^{-\int_{0}^{x} z+\eta(s) \mathrm{d} s} \int_{0}^{x}\left(\mathrm{e}^{-\left(\lambda_{1}+\lambda_{3}\right) s}-\mathrm{e}^{-\left(\lambda_{1}+\lambda_{2}+\lambda_{3}\right) s}\right) \mathrm{d} s \mathrm{~d} x, \\
g_{5}= & \lambda_{1} \int_{0}^{\infty} \eta(x) \mathrm{e}^{-\int_{0}^{x} z+\eta(s) \mathrm{d} s} \int_{0}^{x}\left(\mathrm{e}^{-\left(\lambda_{1}+\lambda_{2}\right) s}-\mathrm{e}^{-\left(\lambda_{1}+\lambda_{2}+\lambda_{3}\right) s}\right) \mathrm{d} s \mathrm{~d} x, \\
g_{6}= & \lambda_{2} \int_{0}^{\infty} \eta(x) \mathrm{e}^{-\int_{0}^{x} z+\eta(s) \mathrm{d} s} \int_{0}^{x}\left(\mathrm{e}^{-\left(\lambda_{1}+\lambda_{2}\right) s}-\mathrm{e}^{-\left(\lambda_{1}+\lambda_{2}+\lambda_{3}\right) s}\right) \mathrm{d} s \mathrm{~d} x \\
& +\lambda_{3} \int_{0}^{\infty} \eta(x) \mathrm{e}^{-\int_{0}^{x} z+\eta(s) \mathrm{d} s} \int_{0}^{x}\left(\mathrm{e}^{-\left(\lambda_{1}+\lambda_{3}\right) s}-\mathrm{e}^{-\left(\lambda_{1}+\lambda_{2}+\lambda_{3}\right) s}\right) \mathrm{d} s \mathrm{~d} x, \\
g_{7}= & \int_{0}^{\infty} \mu_{1}(x) \mathrm{e}^{-\int_{0}^{x} z+\mu_{1}(s) \mathrm{d} s} \mathrm{~d} x \\
g_{8}= & \int_{0}^{\infty} \mu_{2}(x) \mathrm{e}^{-\int_{0}^{x} z+\mu_{2}(s) \mathrm{d} s} \mathrm{~d} x
\end{aligned}
$$




$$
\begin{aligned}
& g_{9}=\int_{0}^{\infty} \mu_{3}(x) \mathrm{e}^{-\int_{0}^{x} z+\mu_{3}(s) \mathrm{d} s} \mathrm{~d} x \\
& g_{10}=\lambda_{3} \int_{0}^{\infty} \mu_{2}(x) \mathrm{e}^{-\int_{0}^{x} z+\mu_{2}(s) \mathrm{d} s} \int_{0}^{x} \mathrm{e}^{-\left(\left(1-d_{1}\right) \lambda_{1}+\lambda_{3}\right) y} \mathrm{~d} y \mathrm{~d} x, \\
& g_{11}=\int_{0}^{\infty} \mu_{2}(x) \mathrm{e}^{-\int_{0}^{y} z+\left(1-d_{1}\right) \lambda_{1}+\lambda_{3}+\mu_{2}(s) \mathrm{d} s} \mathrm{~d} x, \\
& g_{12}=\int_{0}^{\infty} \mu_{3}(x) \mathrm{e}^{-\int_{0}^{y} z+\lambda_{1}\left(1-d_{1}\right)+\lambda_{2}\left(1-d_{2}\right)+\mu_{3}(s) \mathrm{d} s} \mathrm{~d} x
\end{aligned}
$$

then the matrix

$$
T=\left(\begin{array}{cccccccc}
z+\lambda_{1}+\lambda_{2}+\lambda_{3} & 0 & 0 & 0 & -g_{0} & 0 & 0 & 0 \\
-\lambda_{1} & 1 & 0 & 0 & -g_{1} & 0 & 0 & 0 \\
-\lambda_{2} & 0 & 1 & 0 & -g_{2} & -g_{9} & 0 & 0 \\
-\lambda_{3} & 0 & 0 & 1 & -g_{3} & 0 & -g_{7} & -g_{8} \\
0 & -g_{7} & -g_{11} & -g_{12} & 1 & 0 & 0 & 0 \\
0 & 0 & 0 & 0 & -g_{4} & 1 & 0 & 0 \\
0 & 0 & 0 & 0 & -g_{5} & 0 & 1 & 0 \\
0 & 0 & -g_{10} & 0 & -g_{6} & 0 & 0 & 1
\end{array}\right)
$$

is a diagonally dominant matrix about column.

Corollary 2 Let $c=\min \left\{c_{1}, c_{2}, c_{3}, c_{4}\right\}$ and the matrix $T$ be the same as in corollary 1. If $f(z)$ were the determinant of the matrix $T$, then the function $f(z)$ have finite zeros in the region $\{z \in C \mid 0>\operatorname{Re} z>-c\}$.

Furthermore, we need two other lemmas to analyze the spectral distributions of system operator.

Lemma 4 If $z \in\{z \in C \mid 0>\operatorname{Re} z>-c\}$, for each given

$$
\vec{Q}=\left(q_{0}, q_{1}(x), q_{2}(x), q_{3}(x), q_{4}(x, y), q_{5}(x, y), q_{6}(x, y), q_{7}(x), \cdots, q_{16}(x)\right) \in X,
$$

set

$$
\begin{aligned}
f_{1}(y)= & \int_{0}^{y} \mathrm{e}^{-\int_{\tau}^{y} z+\left(1-d_{1}\right) \lambda_{1}+\lambda_{3}+\mu_{2}(s) \mathrm{d} s} q_{2}(\tau) \mathrm{d} \tau \\
f_{2}(y)= & \int_{0}^{y} \mathrm{e}^{-\int_{\tau}^{y} z+\left(1-d_{1}\right) \lambda_{1}+\lambda_{3}+\mu_{2}(s) \mathrm{d} s} \int_{0}^{\infty} \mu_{1}(x) \int_{0}^{x} \mathrm{e}^{-\int_{r}^{x} z+\mu_{1}(s) \mathrm{d} s} q_{4}(r, \tau) \mathrm{d} r \mathrm{~d} x \mathrm{~d} \tau \\
f_{3}(y)= & \int_{0}^{y} \mathrm{e}^{-\int_{\tau}^{y} z+\lambda_{1}\left(1-d_{1}\right)+\lambda_{2}\left(1-d_{2}\right)+\mu_{3}(s) \mathrm{d} s} q_{3}(\tau) \mathrm{d} \tau \\
f_{4}(y)= & \int_{0}^{y} \mathrm{e}^{-\int_{\tau}^{y} z+\lambda_{1}\left(1-d_{1}\right)+\lambda_{2}\left(1-d_{2}\right)+\mu_{3}(s) \mathrm{d} s} \int_{0}^{\infty} \mu_{1}(x) \int_{0}^{x} \mathrm{e}^{-\int_{r}^{x} z+\mu_{1}(s) \mathrm{d} s} q_{5}(r, \tau) \mathrm{d} r \mathrm{~d} x \mathrm{~d} \tau, \\
f_{5}(y)= & \int_{0}^{y} \mathrm{e}^{-\int_{\tau}^{y} z+\lambda_{1}\left(1-d_{1}\right)+\lambda_{2}\left(1-d_{2}\right)+\mu_{3}(s) \mathrm{d} s} \int_{0}^{\infty} \mu_{2}(x) \int_{0}^{x} \mathrm{e}^{-\int_{r}^{x} z+\mu_{2}(s) \mathrm{d} s} q_{6}(r, \tau) \mathrm{d} r \mathrm{~d} x \mathrm{~d} \tau, \\
f_{6}(x)= & \int_{0}^{x} \mathrm{e}^{-\int_{\tau}^{x} z+\mu_{1}(s) \mathrm{d} s} q_{1}(\tau) \mathrm{d} \tau, \\
f_{7}(x)= & \int_{0}^{x} \mathrm{e}^{-\int_{\tau}^{x} z+\lambda_{1}+\lambda_{2}+\lambda_{3}+\eta(s) \mathrm{d} s} q_{7}(\tau) \mathrm{d} \tau
\end{aligned}
$$




$$
\begin{aligned}
& f_{8}(x)=\mathrm{e}^{-\int_{0}^{x} z+\eta(s) \mathrm{d} s} \int_{0}^{x} \mathrm{e}^{\int_{0}^{r} z+\eta(s) \mathrm{d} s} q_{8}(r) \mathrm{d} r, \\
& f_{9}(x)=\lambda_{1} \int_{0}^{x} \int_{0}^{r} \mathrm{e}^{-\left(\lambda_{1}+\lambda_{2}+\lambda_{3}\right)(r-\tau)} \mathrm{e}^{-\int_{\tau}^{x} z+\eta(s) \mathrm{d} s} q_{7}(\tau) \mathrm{d} \tau \mathrm{d} r, \\
& f_{10}(x)=\mathrm{e}^{-\int_{0}^{x} z+\lambda_{1}+\lambda_{3}+\eta(s) \mathrm{d} s} \int_{0}^{x} \mathrm{e}^{\int_{0}^{r} z+\lambda_{1}+\lambda_{3}+\eta(s) \mathrm{d} s} q_{9}(r) \mathrm{d} r, \\
& f_{11}(x)=\lambda_{2} \int_{0}^{x} \int_{0}^{r} \mathrm{e}^{-\lambda_{2}(r-\tau)} \mathrm{e}^{-\int_{\tau}^{x} z+\lambda_{1}+\lambda_{3}+\eta(s) \mathrm{d} s} q_{7}(\tau) \mathrm{d} \tau \mathrm{d} r, \\
& f_{12}(x)=\mathrm{e}^{-\int_{0}^{x} z+\lambda_{1}+\lambda_{2}+\eta(s) \mathrm{d} s} \int_{0}^{x} \mathrm{e}^{\int_{0}^{r} z+\lambda_{1}+\lambda_{2}+\eta(s) \mathrm{d} s} q_{10}(r) \mathrm{d} r, \\
& f_{13}(x)=\lambda_{3} \int_{0}^{x} \int_{0}^{r} \mathrm{e}^{-\lambda_{3}(r-\tau)} \mathrm{e}^{-\int_{\tau}^{x} z+\lambda_{1}+\lambda_{2}+\eta(s) \mathrm{d} s} q_{7}(\tau) \mathrm{d} \tau \mathrm{d} r, \\
& f_{14}(x)=\lambda_{1} \int_{0}^{x} \int_{0}^{u} \mathrm{e}^{-\left(\lambda_{1}+\lambda_{3}\right)(u-r)} \mathrm{e}^{-\int_{r}^{x} z+\eta(s) \mathrm{d} s} q_{9}(r) \mathrm{d} r \mathrm{~d} u, \\
& f_{15}(x)=\lambda_{1} \lambda_{2} \int_{0}^{x} \int_{0}^{u} \int_{0}^{r} \mathrm{e}^{-\left(\lambda_{1}+\lambda_{3}\right)(u-\tau)} \mathrm{e}^{-\lambda_{2}(r-\tau)} \mathrm{e}^{-\int_{\tau}^{x} z+\eta(s) \mathrm{d} s} q_{7}(\tau) \mathrm{d} \tau \mathrm{d} r \mathrm{~d} u, \\
& f_{16}(x)=\int_{0}^{x} \mathrm{e}^{-\int_{r}^{x} z+\mu_{1}(s) \mathrm{d} s} q_{11}(r) \mathrm{d} r, \\
& f_{17}(x)=\lambda_{1} \int_{0}^{x} \int_{0}^{u} \mathrm{e}^{-\left(\lambda_{1}+\lambda_{2}\right)(u-r)} \mathrm{e}^{-\int_{r}^{x} z+\eta(s) \mathrm{d} s} q_{10}(r) \mathrm{d} r \mathrm{~d} u, \\
& f_{18}(x)=\lambda_{1} \lambda_{3} \int_{0}^{x} \int_{0}^{u} \int_{0}^{r} \mathrm{e}^{-\left(\lambda_{1}+\lambda_{2}\right)(u-\tau)} \mathrm{e}^{-\lambda_{3}(r-\tau)} \mathrm{e}^{-\int_{\tau}^{x} z+\eta(s) \mathrm{d} s} q_{7}(\tau) \mathrm{d} \tau \mathrm{d} r \mathrm{~d} u, \\
& f_{19}(x)=\int_{0}^{x} \mathrm{e}^{-\int_{r}^{x} z+\mu_{1}(s) \mathrm{d} s} q_{12}(r) \mathrm{d} r, \\
& f_{20}(x)=\lambda_{2} \int_{0}^{x} \int_{0}^{u} \mathrm{e}^{-\left(\lambda_{1}+\lambda_{2}\right)(u-r)} \mathrm{e}^{-\int_{r}^{x} z+\eta(s) \mathrm{d} s} q_{10}(r) \mathrm{d} r \mathrm{~d} u, \\
& f_{21}(x)=\lambda_{2} \lambda_{3} \int_{0}^{x} \int_{0}^{u} \int_{0}^{r} \mathrm{e}^{-\left(\lambda_{1}+\lambda_{2}\right)(u-\tau)} \mathrm{e}^{-\lambda_{3}(r-\tau)} \mathrm{e}^{-\int_{\tau}^{x} z+\eta(s) \mathrm{d} s} q_{7}(\tau) \mathrm{d} \tau \mathrm{d} r \mathrm{~d} u, \\
& f_{22}(x)=\lambda_{3} \mathrm{e}^{-\int_{0}^{x} z+\eta(s) \mathrm{d} s} \int_{0}^{x} \mathrm{e}^{-\left(\lambda_{1}+\lambda_{3}\right) u} \int_{0}^{u} \mathrm{e}^{\int_{0}^{r} z+\lambda_{1}+\lambda_{3}+\eta(s) \mathrm{d} s} q_{9}(r) \mathrm{d} r \mathrm{~d} u, \\
& f_{23}(x)=\lambda_{2} \lambda_{3} \int_{0}^{x} \int_{0}^{u} \int_{0}^{r} \mathrm{e}^{-\left(\lambda_{1}+\lambda_{3}\right)(u-\tau)} \mathrm{e}^{-\lambda_{2}(r-\tau)} \mathrm{e}^{-\int_{\tau}^{x} z+\eta(s) \mathrm{d} s} q_{7}(\tau) \mathrm{d} \tau \mathrm{d} r \mathrm{~d} u, \\
& f_{24}(x)=\int_{0}^{x} \mathrm{e}^{-\int_{r}^{x} z+\mu_{1}(s) \mathrm{d} s} q_{13}(r) \mathrm{d} r, \\
& f_{25}(x)=\mathrm{e}^{-\int_{0}^{x} z+\mu_{1}(s) \mathrm{d} s} \int_{0}^{x} \mathrm{e}^{\int_{0}^{r} z+\mu_{1}(s) \mathrm{d} s} q_{14}(r) \mathrm{d} r, \\
& f_{26}(x)=\mathrm{e}^{-\int_{0}^{x} z+\mu_{1}(s) \mathrm{d} s} \int_{0}^{x} \mathrm{e}^{\int_{0}^{r} z+\mu_{1}(s) \mathrm{d} s} q_{15}(r) \mathrm{d} r, \\
& f_{27}(x)=\mathrm{e}^{-\int_{0}^{x} z+\mu_{2}(s) \mathrm{d} s} \int_{0}^{x} \mathrm{e}^{\int_{0}^{r} z+\mu_{2}(s) \mathrm{d} s} q_{16}(r) \mathrm{d} r, \\
& f_{28}(x)=\lambda_{3} \mathrm{e}^{-\int_{0}^{x} z+\mu_{2}(s) \mathrm{d} s} \int_{0}^{x} \mathrm{e}^{-\left(\left(1-d_{1}\right) \lambda_{1}+\lambda_{3}\right) y} \int_{0}^{y} \mathrm{e}^{\int_{0}^{\tau} z+\left(1-d_{1}\right) \lambda_{1}+\lambda_{3}+\mu_{2}(s) \mathrm{d} s} q_{2}(\tau) \mathrm{d} \tau \mathrm{d} y, \\
& f_{29}(x)=\lambda_{3} \int_{0}^{x} \int_{0}^{y} \mathrm{e}^{-\left(\left(1-d_{1}\right) \lambda_{1}+\lambda_{3}\right)(y-\tau)} \mathrm{e}^{-\int_{\tau}^{x} z+\mu_{2}(s) \mathrm{d} s} \int_{0}^{\infty} \mu_{1}(u) \int_{0}^{u} \mathrm{e}^{-\int_{r}^{u} z+\mu_{1}(s) \mathrm{d} s} q_{4}(r, \tau) \mathrm{d} r \mathrm{~d} u \mathrm{~d} \tau \mathrm{d} y,
\end{aligned}
$$




$$
\begin{aligned}
& f_{30}(y)=\mathrm{e}^{-\int_{0}^{y} z+\left(1-d_{1}\right) \lambda_{1}+\lambda_{3}+\mu_{2}(s) \mathrm{d} s}, \\
& f_{31}(y)=\mathrm{e}^{-\int_{0}^{y} z+\lambda_{1}\left(1-d_{1}\right)+\lambda_{2}\left(1-d_{2}\right)+\mu_{3}(s) \mathrm{d} s}, \\
& f_{32}(x)=\mathrm{e}^{-\int_{0}^{x} z+\mu_{1}(s) \mathrm{d} s} \\
& f_{33}(x)=\mathrm{e}^{-\int_{0}^{x} z+\lambda_{1}+\lambda_{2}+\lambda_{3}+\eta(s) \mathrm{d} s}, \\
& f_{34}(x)=\lambda_{1} \mathrm{e}^{-\int_{0}^{x} z+\eta(s) \mathrm{d} s} \int_{0}^{x} \mathrm{e}^{-\left(\lambda_{1}+\lambda_{2}+\lambda_{3}\right) s} \mathrm{~d} s, \\
& f_{35}(x)=\mathrm{e}^{-\int_{0}^{x} z+\lambda_{1}+\lambda_{3}+\eta(s) \mathrm{d} s}-\mathrm{e}^{-\int_{0}^{x} z+\lambda_{1}+\lambda_{2}+\lambda_{3}+\eta(s) \mathrm{d} s}, \\
& f_{36}(x)=\mathrm{e}^{-\int_{0}^{x} z+\lambda_{1}+\lambda_{2}+\eta(s) \mathrm{d} s}-\mathrm{e}^{-\int_{0}^{x} z+\lambda_{1}+\lambda_{2}+\lambda_{3}+\eta(s) \mathrm{d} s}, \\
& f_{37}(x)=\lambda_{1} \mathrm{e}^{-\int_{0}^{x} z+\eta(s) \mathrm{d} s} \int_{0}^{x} \mathrm{e}^{-\left(\lambda_{1}+\lambda_{3}\right) s}-\mathrm{e}^{-\left(\lambda_{1}+\lambda_{2}+\lambda_{3}\right) s} \mathrm{~d} s, \\
& f_{38}(x)=\lambda_{1} \mathrm{e}^{-\int_{0}^{x} z+\eta(s) \mathrm{d} s} \int_{0}^{x} \mathrm{e}^{-\left(\lambda_{1}+\lambda_{2}\right) s}-\mathrm{e}^{-\left(\lambda_{1}+\lambda_{2}+\lambda_{3}\right) s} \mathrm{~d} s, \\
& f_{39}(x)=\lambda_{2} \mathrm{e}^{-\int_{0}^{x} z+\eta(s) \mathrm{d} s} \int_{0}^{x} \mathrm{e}^{-\left(\lambda_{1}+\lambda_{2}\right) s}-\mathrm{e}^{-\left(\lambda_{1}+\lambda_{2}+\lambda_{3}\right) s} \mathrm{~d} s+ \\
& \lambda_{3} \mathrm{e}^{-\int_{0}^{x} z+\eta(s) \mathrm{d} s} \int_{0}^{x} \mathrm{e}^{-\left(\lambda_{1}+\lambda_{3}\right) s}-\mathrm{e}^{-\left(\lambda_{1}+\lambda_{2}+\lambda_{3}\right) s} \mathrm{~d} s, \\
& f_{40}(x)=\mathrm{e}^{-\int_{0}^{x} z+\mu_{2}(s) \mathrm{d} s} \\
& f_{41}(x, y)=\mathrm{e}^{-\int_{0}^{x} z+\mu_{1}(s) \mathrm{d} s} \int_{0}^{x} \mathrm{e}^{\int_{0}^{\tau} z+\mu_{1}(s) \mathrm{d} s} q_{4}(\tau, y) \mathrm{d} \tau, \\
& f_{42}(x, y)=\mathrm{e}^{-\int_{0}^{x} z+\mu_{1}(s) \mathrm{d} s} \int_{0}^{x} \mathrm{e}^{\int_{0}^{\tau} z+\mu_{1}(s) \mathrm{d} s} q_{5}(\tau, y) \mathrm{d} \tau, \\
& f_{43}(x, y)=\mathrm{e}^{-\int_{0}^{x} z+\mu_{2}(s) \mathrm{d} s} \int_{0}^{x} \mathrm{e}^{\int_{0}^{\tau} z+\mu_{2}(s) \mathrm{d} s} q_{6}(\tau, y) \mathrm{d} \tau,
\end{aligned}
$$

then the functions $f_{k}(k=1,2, \cdots, 43)$ are absolutely continuous and absolutely integrable.

Lemma 5 If let

$$
\begin{aligned}
e_{0} & =\int_{0}^{\infty} \eta(x) f_{7}(x) \mathrm{d} x \\
e_{1} & =\int_{0}^{\infty} \eta(x)\left[f_{8}(x)+f_{9}(x)\right] \mathrm{d} x \\
e_{2} & =\int_{0}^{\infty} \eta(x)\left[f_{10}(x)+f_{11}(x)\right] \mathrm{d} x+\int_{0}^{\infty} \mu_{1}(x) f_{25}(x) \mathrm{d} x \\
e_{3} & =\int_{0}^{\infty} \eta(x)\left[f_{12}(x)+f_{13}(x)\right] \mathrm{d} x+\int_{0}^{\infty} \mu_{1}(x) f_{26}(x) \mathrm{d} x+\int_{0}^{\infty} \mu_{2}(x)\left[f_{27}(x)+f_{28}(x)+f_{29}(x)\right] \mathrm{d} x \\
e_{4} & =\int_{0}^{\infty} \mu_{1}(x) f_{6}(x) \mathrm{d} x+\int_{0}^{\infty} \mu_{2}(x)\left[f_{1}(x)+f_{2}(x) \mathrm{d} x+\int_{0}^{\infty} \mu_{3}(x)\left[f_{3}(x)+f_{4}(x)+f_{5}(x)\right] \mathrm{d} x\right. \\
e_{5} & =\int_{0}^{\infty} \eta(x)\left[f_{14}(x)+f_{15}(x)+f_{16}(x)\right] \mathrm{d} x \\
e_{6} & =\int_{0}^{\infty} \eta(x)\left[f_{17}(x)+f_{18}(x)+f_{19}(x)\right] \mathrm{d} x \\
e_{7} & =\int_{0}^{\infty} \eta(x)\left[f_{20}(x)+f_{21}(x)+f_{22}(x)+f_{23}(x)+f_{24}(x)\right] \mathrm{d} x
\end{aligned}
$$

in which $f_{k}(x)(k=1,2, \cdots, 29)$ are defined by the above lemma, then for $l=0,1, \cdots, 7, e_{l} \in \mathbb{C}$. 


\section{Spectral analysis of the system operator}

In this section, we shall give the detailed spectral analysis of the system operator with the help of the lemmas and corollaries listed in the previous section. Moreover, several useful corollaries are also obtained.

Theorem 1 Zero is the eigenvalue of system operator A whose geometric multiplicity is one. Furthermore, system operator $A$ has a positive eigenfunction corresponding to eigenvalue zero.

Proof If the equation $A \vec{P}=0$ have nonzero solution in the domain $D(A)$ of $A$, then zero would be the eigenvalue of system operator. However, the equation $A \vec{P}=0$ is equivalent to

$$
\begin{aligned}
& {\left[\lambda_{1}+\lambda_{2}+\lambda_{3}\right] p_{0}=\int_{0}^{\infty} \eta(x) p_{7}(x) \mathrm{d} x} \\
& {\left[\frac{\mathrm{d}}{\mathrm{d} x}+\mu_{1}(x)\right] p_{1}(x)=0} \\
& {\left[\frac{\mathrm{d}}{\mathrm{d} y}+\lambda_{1}+\lambda_{3}+\mu_{2}(y)\right] p_{2}(y)=\int_{0}^{\infty} \mu_{1}(x) p_{4}(x, y) \mathrm{d} x} \\
& {\left[\frac{\mathrm{d}}{\mathrm{d} y}+\lambda_{1}+\lambda_{2}+\mu_{3}(y)\right] p_{3}(y)=\int_{0}^{\infty} \mu_{1}(x) p_{5}(x, y) \mathrm{d} x+\int_{0}^{\infty} \mu_{2}(x) p_{6}(x, y) \mathrm{d} x} \\
& {\left[\frac{\partial}{\partial x}+\mu_{1}(x)\right] p_{4}(x, y)=0} \\
& {\left[\frac{\partial}{\partial x}+\mu_{1}(x)\right] p_{5}(x, y)=0} \\
& {\left[\frac{\partial}{\partial x}+\mu_{2}(x)\right] p_{6}(x, y)=0} \\
& {\left[\frac{\mathrm{d}}{\mathrm{d} x}+\lambda_{1}+\lambda_{2}+\lambda_{3}+\eta(x)\right] p_{7}(x)=0} \\
& {\left[\frac{\mathrm{d}}{\mathrm{d} x}+\eta(x)\right] p_{8}(x)=\lambda_{1} p_{7}(x)} \\
& {\left[\frac{\mathrm{d}}{\mathrm{d} x}+\lambda_{1}+\lambda_{3}+\eta(x)\right] p_{9}(x)=\lambda_{2} p_{7}(x)} \\
& {\left[\frac{\mathrm{d}}{\mathrm{d} x}+\lambda_{1}+\lambda_{2}+\eta(x)\right] p_{10}(x)=\lambda_{3} p_{7}(x)} \\
& {\left[\frac{\mathrm{d}}{\mathrm{d} x}+\eta(x)\right] p_{11}(x)=\lambda_{1} p_{9}(x)} \\
& {\left[\frac{\mathrm{d}}{\mathrm{d} x}+\eta(x)\right] p_{12}(x)=\lambda_{1} p_{10}(x)} \\
& {\left[\frac{\mathrm{d}}{\mathrm{d} x}+\eta(x)\right] p_{13}(x)=\lambda_{2} p_{10}(x)+\lambda_{3} p_{9}(x)} \\
& {\left[\frac{\mathrm{d}}{\mathrm{d} x}+\mu_{1}(x)\right] p_{14}(x)=0} \\
& {\left[\frac{\mathrm{d}}{\mathrm{d} x}+\mu_{1}(x)\right] p_{15}(x)=0} \\
& {\left[\frac{\mathrm{d}}{\mathrm{d} x}+\mu_{2}(x)\right] p_{16}(x)=\lambda_{3} p_{2}(x)}
\end{aligned}
$$


Since the desired nonzero solution $\vec{P}$ belongs to $D(A)$, it must satisfies identities $(25) \sim(31)$. Firstly, it follows from (34) that

$$
p_{1}(x)=p_{1}(0) \mathrm{e}^{-\int_{0}^{x} \mu_{1}(s) \mathrm{d} s}
$$

Solving the equations $(37) \sim(39)$ with the help of (28), we have

$$
\begin{aligned}
& p_{4}(x, y)=\lambda_{1} p_{2}(y) \mathrm{e}^{-\int_{0}^{x} \mu_{1}(s) \mathrm{d} s} \\
& p_{5}(x, y)=\lambda_{1} p_{3}(y) \mathrm{e}^{-\int_{0}^{x} \mu_{1}(s) \mathrm{d} s} \\
& p_{6}(x, y)=\lambda_{2} p_{3}(y) \mathrm{e}^{-\int_{0}^{x} \mu_{2}(s) \mathrm{d} s}
\end{aligned}
$$

Substitute the functions above into the corresponding equations (35) (36), it follows from lemma 1 that

$$
\begin{aligned}
& p_{2}(y)=p_{2}(0) \mathrm{e}^{-\int_{0}^{y} \lambda_{3}+\mu_{2}(s) \mathrm{d} s} \\
& p_{3}(y)=p_{3}(0) \mathrm{e}^{-\int_{0}^{y} \mu_{3}(s) \mathrm{d} s}
\end{aligned}
$$

Secondly, it is easy to see

$$
p_{7}(x)=p_{7}(0) \mathrm{e}^{-\int_{0}^{x} \lambda_{1}+\lambda_{2}+\lambda_{3}+\eta(s) \mathrm{d} s}
$$

Substitute $p_{7}(x)$ into the equations (41) (43) and then solve them with the help of the identity (30), we obtain

$$
\begin{aligned}
& p_{8}(x)=\lambda_{1} p_{7}(0) \mathrm{e}^{-\int_{0}^{x} \eta(s) \mathrm{d} s} \int_{0}^{x} \mathrm{e}^{-\left(\lambda_{1}+\lambda_{2}+\lambda_{3}\right) s} \mathrm{~d} s \\
& p_{9}(x)=\lambda_{2} p_{7}(0) \mathrm{e}^{-\int_{0}^{x} \lambda_{1}+\lambda_{3}+\eta(s) \mathrm{d} s} \int_{0}^{x} \mathrm{e}^{-\lambda_{2} s} \mathrm{~d} s \\
& p_{10}(x)=\lambda_{3} p_{7}(0) \mathrm{e}^{-\int_{0}^{x} \lambda_{1}+\lambda_{2}+\eta(s) \mathrm{d} s} \int_{0}^{x} \mathrm{e}^{-\lambda_{3} s} \mathrm{~d} s
\end{aligned}
$$

Similarly, it follows from the equations $(44) \sim(46)$ that

$$
\begin{aligned}
p_{11}(x)= & \lambda_{1} p_{7}(0) \mathrm{e}^{-\int_{0}^{x} \eta(s) \mathrm{d} s} \int_{0}^{x}\left(\mathrm{e}^{-\left(\lambda_{1}+\lambda_{3}\right) s}-\mathrm{e}^{-\left(\lambda_{1}+\lambda_{2}+\lambda_{3}\right) s}\right) \mathrm{d} s \\
p_{12}(x)= & \lambda_{1} p_{7}(0) \mathrm{e}^{-\int_{0}^{x} \eta(s) \mathrm{d} s} \int_{0}^{x}\left(\mathrm{e}^{-\left(\lambda_{1}+\lambda_{2}\right) s}-\mathrm{e}^{-\left(\lambda_{1}+\lambda_{2}+\lambda_{3}\right) s}\right) \mathrm{d} s \\
p_{13}(x)= & \lambda_{3} p_{7}(0) \mathrm{e}^{-\int_{0}^{x} \eta(s) \mathrm{d} s} \int_{0}^{x}\left(\mathrm{e}^{-\left(\lambda_{1}+\lambda_{3}\right) s}-\mathrm{e}^{-\left(\lambda_{1}+\lambda_{2}+\lambda_{3}\right) s}\right) \mathrm{d} s \\
& +\lambda_{2} p_{7}(0) \mathrm{e}^{-\int_{0}^{x} \eta(s) \mathrm{d} s} \int_{0}^{x}\left(\mathrm{e}^{-\left(\lambda_{1}+\lambda_{2}\right) s}-\mathrm{e}^{-\left(\lambda_{1}+\lambda_{2}+\lambda_{3}\right) s}\right) \mathrm{d} s
\end{aligned}
$$

At last, solving the equations $(47) \sim(49)$ leads to

$$
\begin{aligned}
& p_{14}(x)=p_{14}(0) \mathrm{e}^{-\int_{0}^{x} \mu_{1}(s) \mathrm{d} s} \\
& p_{15}(x)=p_{15}(0) \mathrm{e}^{-\int_{0}^{x} \mu_{1}(s) \mathrm{d} s} \\
& p_{16}(x)=p_{16}(0) \mathrm{e}^{-\int_{0}^{x} \mu_{2}(s) \mathrm{d} s}+\lambda_{3} p_{2}(0) \mathrm{e}^{-\int_{0}^{x} \mu_{2}(s) \mathrm{d} s} \int_{0}^{x} \mathrm{e}^{-\lambda_{3} s} \mathrm{~d} s
\end{aligned}
$$

Substitute the above functions $p_{j}(x)(j=1,2,3,7, \cdots, 16)$ into the identities $(25) \sim(27),(29)$, (31) and (33), we will obtain a homogeneous linear equations on $p_{0}, p_{1}(0), p_{2}(0), p_{3}(0), p_{7}(0)$, 
$p_{14}(0), p_{15}(0)$ and $p_{16}(0)$

$$
\begin{aligned}
& -\left(\lambda_{1}+\lambda_{2}+\lambda_{3}\right) p_{0}+a_{1} p_{7}(0)=0, \\
& \lambda_{1} p_{0}-p_{1}(0)+a_{2} p_{7}(0)=0, \\
& \lambda_{3} p_{0}+b_{1} p_{2}(0)-p_{3}(0)+a_{4} p_{7}(0)+p_{15}(0)+p_{16}(0)=0, \\
& p_{1}(0)+b_{2} p_{2}(0)+p_{3}(0)-p_{7}(0)=0, \\
& a_{5} p_{7}(0)-p_{14}(0)=0, \\
& a_{6} p_{7}(0)-p_{15}(0)=0, \\
& \left(a_{7}+a_{8}\right) p_{7}(0)-p_{16}(0)=0,
\end{aligned}
$$

in which the notations $a_{i}(i=1, \cdots, 8)$ and $b_{j}(j=1,2)$ are given in lemma 2 . If let

$$
B=\left(\begin{array}{cccccccc}
-\left(\lambda_{1}+\lambda_{2}+\lambda_{3}\right) & 0 & 0 & 0 & a_{1} & 0 & 0 & 0 \\
\lambda_{1} & -1 & 0 & 0 & a_{2} & 0 & 0 & 0 \\
\lambda_{2} & 0 & -1 & 0 & a_{3} & 1 & 0 & 0 \\
\lambda_{3} & 0 & b_{1} & -1 & a_{4} & 0 & 1 & 1 \\
0 & 1 & b_{2} & 1 & -1 & 0 & 0 & 0 \\
0 & 0 & 0 & 0 & a_{5} & -1 & 0 & 0 \\
0 & 0 & 0 & 0 & a_{6} & 0 & -1 & 0 \\
0 & 0 & 0 & 0 & a_{7}+a_{8} & 0 & 0 & -1
\end{array}\right),
$$

then the coefficients matrix of the equations is the matrix $B$. Since the sum of every column of $B$ is zero by the arguments of lemma 2 , the determinant of the matrix $B$ is zero. Thus, there exists nonzero solution to the equations. This means the nonzero solution to the equation $A \vec{P}=0$ exists, so zero is an eigenvalue of the operator $A$. Moreover, it follows from the equations that

$$
\begin{aligned}
p_{0}=a_{1} & \left(\lambda_{1}+\lambda_{2}+\lambda_{3}\right)^{-1} p_{7}(0) \\
p_{1}(0)= & {\left[\lambda_{1} a_{1}\left(\lambda_{1}+\lambda_{2}+\lambda_{3}\right)^{-1}+a_{2}\right] p_{7}(0) } \\
p_{2}(0)= & {\left[\lambda_{2} a_{1}\left(\lambda_{1}+\lambda_{2}+\lambda_{3}\right)^{-1}+a_{3}+a_{5}\right] p_{7}(0) } \\
p_{3}(0)= & {\left[\lambda_{3} a_{1}\left(\lambda_{1}+\lambda_{2}+\lambda_{3}\right)^{-1}+a_{4}+a_{6}+a_{7}+a_{8}\right] p_{7}(0) } \\
& +b_{1}\left[\lambda_{2} a_{1}\left(\lambda_{1}+\lambda_{2}+\lambda_{3}\right)^{-1}+a_{3}+a_{5}\right] p_{7}(0) \\
p_{14}(0)= & a_{5} p_{7}(0) \\
p_{15}(0)= & a_{6} p_{7}(0) \\
p_{16}(0)= & \left(a_{7}+a_{8}\right) p_{7}(0)
\end{aligned}
$$

which show the geometric multiplicity of the eigenvalue zero is one. Thus the first statement of the theorem is right. The second statement is easy to be verified by setting $p_{7}(0)=1$. The proof of the theorem is complete.

Moreover, it is not hard to know the system operator $A$ is densely defined and closed by direct verification. We do not want to give the proofs of both properties because the verification is trivial. For more information on the denseness and closeness of the similar operator, you can 
refer to [22]. With the help of these basic properties, we can present the following spectral distribution of the system operator.

Theorem 2 The spectral set $\sigma(A)$ of the system operator $A$ lies in left half-plane $\{z \in$ $\mathbb{C} \mid \operatorname{Re} z<0\}$ and zero is the unique point spectrum on the imaginary axis.

Proof To show the arguments of the theorem 2, if let $\rho(A)$ be the resolvent set of the operator $A$, then it is sufficient to show $T:=\{z \in \mathbb{C} \mid \operatorname{Re} z>0$ or $z=i a, a \neq 0\} \subset \rho(A)$. To this end, for each given $\vec{Q} \in X$ and $z \in T$, consider the equation $(z I-A) \vec{P}=\vec{Q}$, which is equivalent to

$$
\begin{aligned}
& {\left[z+\lambda_{1}+\lambda_{2}+\lambda_{3}\right] p_{0}-\int_{0}^{\infty} \eta(x) p_{7}(x) \mathrm{d} x=q_{0},} \\
& p_{1}^{\prime}(x)=-\left[z+\mu_{1}(x)\right] p_{1}(x)+q_{1}(x), \\
& p_{2}^{\prime}(y)=-\left[z+\lambda_{1}+\lambda_{3}+\mu_{2}(y)\right] p_{2}(y)+\int_{0}^{\infty} \mu_{1}(x) p_{4}(x, y) \mathrm{d} x+q_{2}(y), \\
& p_{3}^{\prime}(y)=-\left[z+\lambda_{1}+\lambda_{2}+\mu_{3}(y)\right] p_{3}(y)+\int_{0}^{\infty} \mu_{1}(x) p_{5}(x, y) \mathrm{d} x+\int_{0}^{\infty} \mu_{2}(x) p_{6}(x, y) \mathrm{d} x+q_{3}(y), \\
& \frac{\partial p_{4}(x, y)}{\partial x}=-\left[z+\mu_{1}(x)\right] p_{4}(x, y)+q_{4}(x, y), \\
& \frac{\partial p_{5}(x, y)}{\partial x}=-\left[z+\mu_{1}(x)\right] p_{5}(x, y)+q_{5}(x, y), \\
& \frac{\partial p_{6}(x, y)}{\partial x}=-\left[z+\mu_{2}(x)\right] p_{6}(x, y)+q_{6}(x, y), \\
& p_{7}^{\prime}(x)=-\left[z+\lambda_{1}+\lambda_{2}+\lambda_{3}+\eta(x)\right] p_{7}(x)+q_{7}(x), \\
& p_{8}^{\prime}(x)=-[z+\eta(x)] p_{8}(x)+\lambda_{1} p_{7}(x)+q_{8}(x), \\
& p_{9}^{\prime}(x)=-\left[z+\lambda_{1}+\lambda_{3}+\eta(x)\right] p_{9}(x)+\lambda_{2} p_{7}(x)+q_{9}(x), \\
& p_{10}^{\prime}(x)=-\left[z+\lambda_{1}+\lambda_{2}+\eta(x)\right] p_{10}(x)+\lambda_{3} p_{7}(x)+q_{10}(x), \\
& p_{11}^{\prime}(x)=-[z+\eta(x)] p_{11}(x)+\lambda_{1} p_{9}(x)+q_{11}(x), \\
& p_{12}^{\prime}(x)=-[z+\eta(x)] p_{12}(x)+\lambda_{1} p_{10}(x)+q_{12}(x), \\
& p_{13}^{\prime}(x)=-[z+\eta(x)] p_{13}(x)+\lambda_{2} p_{10}(x)+\lambda_{3} p_{9}(x)+q_{13}(x), \\
& p_{14}^{\prime}(x)=-\left[z+\mu_{1}(x)\right] p_{14}(x)+q_{14}(x), \\
& p_{15}^{\prime}(x)=-\left[z+\mu_{1}(x)\right] p_{15}(x)+q_{15}(x), \\
& p_{16}^{\prime}(x)=-\left[z+\mu_{2}(x)\right] p_{16}(x)+\lambda_{3} p_{2}(x)+q_{16}(x) .
\end{aligned}
$$

Firstly, solving the equations above by the functions defined in lemma 4, we have

$$
\begin{aligned}
& p_{1}(x)=p_{1}(0) f_{32}(x)+f_{6}(x), \\
& p_{2}(y)=p_{2}(0) f_{30}(y)+f_{1}(y)+f_{2}(y), \\
& p_{3}(y)=p_{2}(0) f_{31}(y)+f_{3}(y)+f_{4}(y)+f_{5}(y), \\
& p_{4}(x, y)=\lambda_{1} p_{2}(y) f_{32}(x)+f_{41}(x, y), \\
& p_{5}(x, y)=\lambda_{1} p_{3}(y) f_{32}(x)+f_{42}(x, y), \\
& p_{6}(x, y)=\lambda_{2} p_{3}(y) f_{40}(x)+f_{43}(x, y), \\
& p_{7}(x)=p_{7}(0) f_{33}(x)+f_{7}(x)
\end{aligned}
$$




$$
\begin{aligned}
& p_{8}(x)=p_{7}(0) f_{34}(x)+f_{8}(x)+f_{9}(x), \\
& p_{9}(x)=p_{7}(0) f_{35}(x)+f_{10}(x)+f_{11}(x), \\
& p_{10}(x)=p_{7}(0) f_{36}(x)+f_{12}(x)+f_{13}(x), \\
& p_{11}(x)=p_{7}(0) f_{37}(x)+f_{14}(x)+f_{15}(x)+f_{16}(x), \\
& p_{12}(x)=p_{7}(0) f_{38}(x)+f_{17}(x)+f_{18}(x)+f_{19}(x), \\
& p_{13}(x)=p_{7}(0) f_{39}(x)+f_{20}(x)+f_{21}(x)+f_{22}(x)+f_{23}(x)+f_{24}(x), \\
& p_{14}(x)=p_{14}(0) f_{32}(x)+f_{25}(x), \\
& p_{15}(x)=p_{15}(0) f_{32}(x)+f_{26}(x), \\
& p_{16}(x)=p_{16}(0) f_{40}(x)+f_{27}(x)+f_{28}(x)+f_{29}(x) .
\end{aligned}
$$

Substitute the above functions into corresponding boundary conditions $(25) \sim(27),(29),(31)$ and the first equation, inhomogeneous linear equations on $p_{0}, p_{1}(0), p_{2}(0), p_{3}(0), p_{7}(0), p_{14}(0)$, $p_{15}(0), p_{16}(0)$

$$
\begin{aligned}
\left(z+\lambda_{1}+\lambda_{2}+\lambda_{3}\right) p_{0}-g_{0} p_{7}(0) & =e_{0}+q_{0} \\
-\lambda_{1} p_{0}+p_{1}(0)-g_{1} p_{7}(0) & =e_{1} \\
-\lambda_{2} p_{0}+p_{2}(0)-g_{2} p_{7}(0)-g_{9} p_{14}(0) & =e_{2} \\
-\lambda_{3} p_{0}+p_{3}(0)-g_{3} p_{7}(0)-g_{7} p_{15}(0)-g_{8} p_{16}(0) & =e_{3} \\
-g_{7} p_{1}(0)-g_{11} p_{2}(0)-g_{12} p_{3}(0)+p_{7}(0) & =e_{4} \\
-g_{4} p_{7}(0)+p_{14}(0) & =e_{5} \\
-g_{5} p_{7}(0)+p_{15}(0) & =e_{6} \\
-g_{10} p_{2}(0)-g_{6} p_{7}(0)+p_{16}(0) & =e_{7}
\end{aligned}
$$

are obtained with the help of the notations in corollary 2 and lemma 5 . The coefficient matrix of the equations (73) (80) is just the matrix $T$ given in corollary 2 , which tells us the matrix $T$ is a diagonally dominant matrix about column, so it is nonsingular (see page 184 of [21]). According to lemma $5, e_{l}(l=0,1, \cdots, 7)$ belong to the complex number set $\mathbb{C}$. These statements imply the unique solution to the equations $(73) \sim(80)$ exists. Since $p_{k}(x)(k=1,2,3,7, \cdots, 16)$ and $p_{j}(x, y)(j=4,5,6)$ are uniquely determined by $p_{1}(0), p_{2}(0), p_{3}(0), p_{7}(0), p_{14}(0), p_{15}(0), p_{16}(0)$, the unique solution to the equation $(z I-A) \vec{P}=\vec{Q}$ exists in the domain $D(A)$. This means the operator $z I-A$ be bijective, which is equivalent to its inverse $(z I-A)^{-1}$ exists. $(z I-A)^{-1}$ is closed because the operator $z I-A$ is closed. The classical closed graph theorem is applied to show the boundedness of the inverse operator $(z I-A)^{-1}$, that is to say $z \in \rho(A)$. Thus we complete the proof of the theorem.

Recall that the spectral bound $s(T)$ of an unbounded operator $T$ is the quantity $\sup \{\operatorname{Re} \lambda$ : $\lambda \in \sigma(T)\}$. Thus, theorem 1 and theorem 2 imply the spectral bound $s(A)$ of the system operator $A$ is zero. Moreover, the proof of the theorem 2 implies the following corollary is true.

Corollary 3 There exists a positive constant $\delta$ such that $\{z \mid z \in \mathbb{C}, \operatorname{Re} z>-\delta, z \neq 0\} \subset$ $\rho(A)$.

Proof According to the proof of the theorem 2, we know, for $z \in \mathbb{C}, \operatorname{Re} z>-c, z \in \rho(A)$ if and only if $f(z) \neq 0$. Notice that $f(z)$ is given in proof of corollary 2 and it shows the analytic 
function $f(z)$ has finite zeros in the strip $\{z \in \mathbb{C} \mid-c<\operatorname{Re} z<0\}$. If let $z_{1}, z_{2}, \cdots, z_{n}$ be the zeros of $f(z)$ and $\delta=-\max \left\{\operatorname{Re}_{i} \mid 1 \leq i \leq n\right\}$, then we would have $\{z \mid z \in \mathbb{C}, \operatorname{Re} z>-\delta, z \neq$ $0\} \subset \rho(A)$.

To give the next corollary, we need some notations on the state space $X$. For $\vec{P} \in X$, we assume

$$
\vec{P}=\left(p_{0}, p_{1}(x), p_{2}(x), p_{3}(x), p_{4}(x, y), p_{5}(x, y), p_{6}(x, y), p_{7}(x), \cdots, p_{16}(x)\right)
$$

and call $\vec{P}$ positive (in symbol $0 \leq \vec{P}$ ) if $0 \leq p_{0}$ and

$$
\begin{gathered}
0 \leq p_{i}(x)(i=1,2,3,7, \cdots, 16) \text { for almost all } x \in \mathbb{R}^{+}, \\
0 \leq p_{k}(x, y)(k=4,5,6) \text { for almost all }(x, y) \in \mathbb{R}^{+} \times \mathbb{R}^{+} .
\end{gathered}
$$

To indicate that $0 \leq \vec{P}$ and $0 \neq \vec{P}$ we use the notation $0<\vec{P}$. We call a vector function $\vec{P}$ is strictly positive (in symbol $0 \ll \vec{P}$ ) if $0<p_{0}$ and

$$
\begin{aligned}
& 0<p_{i}(x)(i=1,2,3,7, \cdots, 16) \text { for almost all } x \in \mathbb{R}^{+}, \\
& 0<p_{k}(x, y)(k=4,5,6) \text { for almost all }(x, y) \in \mathbb{R}^{+} \times \mathbb{R}^{+} .
\end{aligned}
$$

In fact, the above notations and concepts are the terminologies of Banach lattice. You can refer to [23-25] for detailed information. Thus, we have the following corollary by proof of the theorem 2 .

Corollary 4 If $\vec{Q} \in X$ and $\vec{Q}>0$, then $R(\lambda, A) \vec{Q} \gg 0$ for $\lambda>s(A)=0$.

Proof According to the proof of the theorem 2, $R(\lambda, A) \vec{Q}$ exists uniquely for each given $\vec{Q} \in X$. It is easy to see if $p_{0}, p_{1}(0), p_{2}(0), p_{3}(0), p_{7}(0), p_{14}(0), p_{15}(0), p_{16}(0)$ are all positive, then $R(\lambda, A) \vec{Q} \gg 0$. However, from the equations $(73) \sim(80)$, if $p_{7}(0)$ is positive, then $p_{0}, p_{1}(0)$, $p_{2}(0), p_{3}(0), p_{14}(0), p_{15}(0), p_{16}(0)$ are also. Thus, to complete the proof of this corollary, it is sufficient to show $p_{7}(0)>0$. To this end, let

$$
T_{5}=\left(\begin{array}{cccccccc}
\lambda+\lambda_{1}+\lambda_{2}+\lambda_{3} & 0 & 0 & 0 & e_{0}+q_{0} & 0 & 0 & 0 \\
-\lambda_{1} & 1 & 0 & 0 & e_{1} & 0 & 0 & 0 \\
-\lambda_{2} & 0 & 1 & 0 & e_{2} & -g_{9} & 0 & 0 \\
-\lambda_{3} & 0 & 0 & 1 & e_{3} & 0 & -g_{7} & -g_{8} \\
0 & -g_{7} & -g_{11} & -g_{12} & e_{4} & 0 & 0 & 0 \\
0 & 0 & 0 & 0 & e_{5} & 1 & 0 & 0 \\
0 & 0 & 0 & 0 & e_{6} & 0 & 1 & 0 \\
0 & 0 & -g_{10} & 0 & e_{7} & 0 & 0 & 1
\end{array}\right),
$$

then the Cramer's rule is applied to the equations $(73) \sim(80)$ results in $p_{7}(0)=\frac{\left|T_{5}\right|}{|T|}$, in which

$$
\begin{aligned}
|T|= & \left(\lambda+\lambda_{1}+\lambda_{2}+\lambda_{3}\right)\left[1-g_{12}\left(g_{3}+g_{6} g_{8}+g_{5} g_{7}\right)-g_{1} g_{7}\right. \\
& \left.-\left(g_{2}+g_{9} g_{4}\right)\left(g_{11}+g_{8} g_{10} g_{12}\right)\right]-g_{0}\left[\lambda_{2}\left(g_{11}+g_{8} g_{10} g_{12}\right)+\left(\lambda_{3} g_{12}+\lambda_{1} g_{7}\right)\right]
\end{aligned}
$$

is given in proof of the corollary 2. Finally, for $\lambda>s(A)=0$, according to the results of corollary 2 we know $0<g_{k}<1(k=7,8,9,12), 0<\sum_{i=0}^{6}\left|g_{i}\right|<1$ and $0<g_{10}+g_{11}<1$. This means $|T|>0$. Comparing the matrix $T_{5}$ with the matrix $T$, it is easy to obtain the determinant of matrix $T_{5}$ 


$$
\begin{aligned}
\left|T_{5}\right|= & \left(\lambda+\lambda_{1}+\lambda_{2}+\lambda_{3}\right)\left[e_{4}+g_{12}\left(e_{3}+e_{7} g_{8}+e_{6} g_{7}\right)+e_{1} g_{7}+\left(e_{2}+g_{9} e_{5}\right)\right. \\
& \left.\times\left(g_{11}+g_{8} g_{10} g_{12}\right)\right]+\left(e_{0}+q_{0}\right)\left[\lambda_{2}\left(g_{11}+g_{8} g_{10} g_{12}\right)+\left(\lambda_{3} g_{12}+\lambda_{1} g_{7}\right)\right] .
\end{aligned}
$$

Since one of the right terms of the above identity must be positive and the other terms are nonnegative, $\left|T_{5}\right|$ is greater than 0 . Thus, $p_{7}(0)>0$ due to $p_{7}(0)=\frac{\left|T_{5}\right|}{|T|}$. Thus the proof of the corollary is complete.

\section{The well-posedness and stability of the system}

By Theorem II 6.7 and Definition II 6.8 of [25], we know that the well-posedness of the system (32) is equivalent to the operator $A$ generating a $C_{0}$ semigroup $T(t)$ on $X$. In fact, the $C_{0}$ semigroup $T(t)$ generated by the operator $A$ is even positive, contractive and irreducible since we have the following results.

Theorem 3 The operator $A$ is a dispersive operator in $X$.

Proof We denote by $X_{+}$and $[\vec{P}]^{+}$the positive cone of the state space and the positive part of the vector $\vec{P}$ in $X$, respectively. For arbitrary $\vec{P} \in D(A)$, set

$$
\begin{gathered}
\phi=\left\|[\vec{P}]^{+}\right\|\left(\operatorname{sign}\left(p_{0}\right), \operatorname{sign}\left(p_{1}(x)\right), \operatorname{sign}\left(p_{2}(x)\right), \operatorname{sign}\left(p_{3}(x)\right), \operatorname{sign}\left(p_{4}(x, y)\right),\right. \\
\left.\operatorname{sign}\left(p_{5}(x, y)\right), \operatorname{sign}\left(p_{6}(x, y)\right), \operatorname{sign}\left(p_{7}(x)\right), \cdots, \operatorname{sign}\left(p_{16}(x)\right)\right),
\end{gathered}
$$

in which $\operatorname{sign}(a)$ is the sign of the number $a$. Then $\phi \in X_{+}^{*}$ and the identities $\|\phi\|^{2}=\left\|[\vec{P}]^{+}\right\|^{2}=$ $(\vec{P}, \phi)$ obviously hold. Now we verify $(A \vec{P}, \phi) \leq 0$. By the definition of the operator $A$, it is easy to see

$$
\begin{aligned}
& \left\|[\vec{P}]^{+}\right\|^{-1}(A \vec{P}, \phi) \\
\leq & -\left[\lambda_{1}+\lambda_{2}+\lambda_{3}\right]\left[p_{0}\right]^{+}-\int_{0}^{\infty} \mu_{1}(x)\left[p_{1}(x)\right]^{+} \mathrm{d} x \\
& -\int_{0}^{\infty}\left(\lambda_{1}+\mu_{2}(y)\right)\left[p_{2}(y)\right]^{+} \mathrm{d} y-\int_{0}^{\infty}\left(\lambda_{1}+\lambda_{2}+\mu_{3}(y)\right)\left[p_{3}(y)\right]^{+} \mathrm{d} y \\
& +\left[p_{1}(0)\right]^{+}+\left[p_{2}(0)\right]^{+}+\left[p_{3}(0)\right]^{+}+\left[p_{7}(0)\right]^{+}+\left[p_{14}(0)\right]^{+}+\left[p_{15}(0)\right]^{+}+\left[p_{16}(0)\right]^{+} \\
& -\int_{0}^{\infty} \eta(x) \sum_{i=8}^{13}\left[p_{i}(x)\right]^{+} \mathrm{d} x+\int_{0}^{\infty}\left[p_{4}(0, y)\right]^{+}+\left[p_{5}(0, y)\right]^{+}+\left[p_{6}(0, y)\right]^{+} \mathrm{d} y \\
& -\int_{0}^{\infty} \mu_{1}(x)\left(\left[p_{14}(x)\right]^{+}+\left[p_{15}(x)\right]^{+}\right)-\mu_{2}(x)\left[p_{16}(x)\right]^{+} \mathrm{d} x .
\end{aligned}
$$

It follows from the boundary conditions $(25) \sim(31)$ that

$$
\begin{aligned}
& {\left[p_{1}(0)\right]^{+} \leq \lambda_{1}\left[p_{0}\right]^{+}+\int_{0}^{\infty} \eta(x)\left[p_{8}(x)\right]^{+} \mathrm{d} x,} \\
& {\left[p_{2}(0)\right]^{+} \leq \lambda_{2}\left[p_{0}\right]^{+}+\int_{0}^{\infty} \eta(x)\left[p_{9}(x)\right]^{+} \mathrm{d} x+\int_{0}^{\infty} \mu_{1}(x)\left[p_{14}(x)\right]^{+} \mathrm{d} x,} \\
& {\left[p_{3}(0)\right]^{+} \leq \lambda_{3}\left[p_{0}\right]^{+}+\int_{0}^{\infty} \eta(x)\left[p_{10}(x)\right]^{+} \mathrm{d} x+\int_{0}^{\infty} \mu_{1}(x)\left[p_{15}(x)\right]^{+} \mathrm{d} x+\int_{0}^{\infty} \mu_{2}(x)\left[p_{16}(x)\right]^{+} \mathrm{d} x,} \\
& {\left[p_{4}(0, y)\right]^{+}=\lambda_{1}\left[p_{2}(y)\right]^{+}, \quad p_{5}(0, y)=\lambda_{1}\left[p_{3}(y)\right]^{+}, \quad\left[p_{6}(0, y)\right]^{+}=\lambda_{2}\left[p_{3}(y)\right]^{+},} \\
& p_{7}(0) \leq \int_{0}^{\infty} \mu_{1}(x)\left[p_{1}(x)\right]^{+} \mathrm{d} x+\int_{0}^{\infty} \mu_{2}(x)\left[p_{2}(x)\right]^{+} \mathrm{d} x+\int_{0}^{\infty} \mu_{3}(x)\left[p_{3}(x)\right]^{+} \mathrm{d} x,
\end{aligned}
$$




$$
\begin{aligned}
& {\left[p_{14}(0)\right]^{+} \leq \int_{0}^{\infty} \eta(x)\left[p_{11}(x)\right]^{+} \mathrm{d} x,} \\
& {\left[p_{15}(0)\right]^{+} \leq \int_{0}^{\infty} \eta(x)\left[p_{12}(x)\right]^{+} \mathrm{d} x,} \\
& {\left[p_{16}(0)\right]^{+} \leq \int_{0}^{\infty} \eta(x)\left[p_{13}(x)\right]^{+} \mathrm{d} x .}
\end{aligned}
$$

If substitute them into the original inequality, we obtain $(A \vec{P}, \phi) \leq 0$. The operator $A$ is therefore dispersive by the definition of the dispersive operator in [23].

Now we can obtain one of the main results of this note.

Theorem 4 The operator $A$ generates some positive $C_{0}$ semigroups $T(t)$, which is contractive and irreducible, on Banach lattice $X$. Therefore, the unique non-negative solution $\vec{p}(\cdot, t)$ of the system $(1) \sim(24)$ exists and it is given by $T(t) \vec{p}(0)$.

Proof According to Philips Theorem in the positive semigroup theory (see [23]), $A$ generates a positive $C_{0}$-semigroup of contractions if and only if $A$ is a dispersive and the operator $I-A$ is surjective. Since theorem 2 has asserted that 1 belongs to the resolvent set of the operator $A$ and theorem 3 show that $A$ is a dispersive, we obtain the semigroup $T(t)$ generated by system operator $A$ is positive and contractive. Thus the equations (1) (24) have a unique solution by well known result in the theory of the $C_{0}$-semigroup and it could be expressed as $T(t) \vec{p}(0)$. Moreover, the unique solution $T(t) \vec{p}(0)$ is non-negative since the inial value $\vec{p}(0)$ in (32) is non-negative and the semigroup $T(t)$ is positive. At last, it follows the corollary 4 and the definition VI 3.1 in [25] that the semigroup $T(t)$ is irreducible. The proof of the theorem is complete.

Furthermore, we can obtain the asymptotic stability of the the system (32). To this end, we have to show zero is the simple eigenvalue of the system operator $A$. This result is given in the following theorem.

Theorem 5 Zero is the simple eigenvalue of the system operator A.

Proof By the theory of the functional analysis, we know that the dual space of the state space $X$ is $X^{*}=\mathbb{R} \times\left(L^{\infty}\left(\mathbb{R}^{+}\right)\right)^{3} \times\left(L^{\infty}\left(\mathbb{R}^{+} \times \mathbb{R}^{+}\right)\right)^{3} \times\left(L^{\infty}\left(\mathbb{R}^{+}\right)\right)^{10}$. For $\vec{Q} \in X^{*}$, the norm of $\vec{Q}$ is given by

$$
\|\vec{Q}\|=\max \left\{\left|q_{0}\right|, \sup _{x \in \mathbb{R}^{+}} q_{i}(x), \sup _{(x, y) \in \mathbb{R}^{+} \times \mathbb{R}^{+}} q_{j}(x, y), i=1,2,3,7, \cdots, 16, j=4,5,6\right\} .
$$

If let $\vec{Q}=(1,1, \cdots, 1) \in X^{*}$, then $\vec{Q} \in D\left(A^{*}\right)$ and $A^{*} \vec{Q}=0$. This means that zero is the eigenvalue of the operator $A^{*}$ and $\vec{Q}$ is the corresponding eigenfunction. Let $\widehat{P}$ be the nonnegative eigenfunction corresponding to eigenvalue 0 . Since $(\widehat{P}, \vec{Q})=\|\widehat{P}\| \neq 0$, we therefore deduce that zero is the simple eigenvalue of the system operator $A$ by similar argument in the theorem 4.1.2 of reference [19]. Since it is easy to obtain the dual operator of the system operator $A$ as follows,

$$
D\left(A^{*}\right)=\left\{\begin{array}{l|l}
\vec{Q} \in X^{*} & \begin{array}{l}
q_{i}^{\prime}(x) \in L^{\infty}\left(\mathbb{R}^{+}\right), \frac{\partial q_{j}(x, y)}{\partial x} \in L^{\infty}\left(\mathbb{R}^{+} \times \mathbb{R}^{+}\right), q_{i}(x) \text { and } q_{j}(x, y) \text { are } \\
\text { absolutely continuous and they satisfy } q_{i}(\infty), q_{j}(\infty, y) \in \mathbb{R}, i= \\
1,2,3,7, \cdots, 16, j=4,5,6 .
\end{array}
\end{array}\right\}
$$




$$
A^{*} \vec{Q}=\left(\begin{array}{c}
\lambda_{1} q_{1}(0)+\lambda_{2} q_{2}(0)+\lambda_{3} q_{3}(0)-\left(\lambda_{1}+\lambda_{2}+\lambda_{3}\right) q_{0} \\
q_{1}^{\prime}(y)+\left[q_{7}(0)-q_{1}(x)\right] \mu_{1}(x) \\
q_{2}^{\prime}(y)+\lambda_{1} q_{4}(0, y)+q_{7}(0) \mu_{2}(y)+\lambda_{3} q_{16}(y)-\left(\lambda_{1}+\lambda_{3}+\mu_{2}(y)\right) q_{2}(y) \\
q_{3}^{\prime}(y)+\lambda_{1} q_{5}(0, y)+\lambda_{2} q_{6}(0, y)+q_{7}(0) \mu_{3}(y)-\left(\lambda_{1}+\lambda_{2}+\mu_{3}(y)\right) q_{3}(y) \\
\frac{\partial q_{4}(x, y)}{\partial x}+\left[q_{2}(y)-q_{4}(x, y)\right] \mu_{1}(x) \\
\frac{\partial q_{5}(x, y)}{\partial x}+\left[q_{3}(y)-q_{5}(x, y)\right] \mu_{1}(x) \\
\frac{\partial q_{6}(x, y)}{\partial x}+\left[q_{3}(y)-q_{6}(x, y)\right] \mu_{2}(x) \\
q_{7}^{\prime}(x)+q_{0} \eta(x)+\lambda_{1} q_{8}(x)+\lambda_{2} q_{9}(x)+\lambda_{3} q_{10}(x)-\left(\lambda_{1}+\lambda_{2}+\lambda_{3}+\eta(x)\right) q_{7}(x) \\
q_{8}^{\prime}(x)+\left(q_{1}(0)-q_{8}(x)\right) \eta(x) \\
q_{9}^{\prime}(x)+q_{2}(0) \eta(x)+\lambda_{1} q_{11}(x)+\lambda_{3} q_{13}(x)-\left(\lambda_{1}+\lambda_{3}+\eta(x)\right) q_{9}(x) \\
q_{10}^{\prime}(x)+q_{3}(0) \eta(x)+\lambda_{1} q_{12}(x)+\lambda_{2} q_{13}(x)-\left(\lambda_{1}+\lambda_{2}+\eta(x)\right) q_{10}(x) \\
q_{11}^{\prime}(x)+\left[q_{14}(0)-q_{11}(x)\right] \eta(x) \\
q_{12}^{\prime}(x)+\left[q_{14}(0)-q_{12}(x)\right] \eta(x) \\
q_{13}^{\prime}(x)+\left[q_{16}(0)-q_{13}(x)\right] \eta(x) \\
q_{14}^{\prime}(x)+\left[q_{2}(0)-q_{14}(x)\right] \mu_{1}(x) \\
q_{15}^{\prime}(x)+\left[q_{3}(0)-q_{15}(x)\right] \mu_{1}(x) \\
q_{16}^{\prime}(x)+\left[q_{3}(0)-q_{16}(x)\right] \mu_{2}(x)
\end{array}\right)
$$

From the definition of the strictly dominant eigenvalue in [15], corollary 3 and the above theorem, we can get the following result.

Corollary 5 Zero is a strictly dominant eigenvalue of the system operator A.

Now, we show another main result of this note, that is the steady-state solution to the equations (1) (24) is existed and the dynamic solution converges to the steady-state solution in the norm topology of state space $X$. We elaborate on this property in following theorem. Indeed, the following theorem is a direct consequence of the theorem 2, theorem 5 and lemma 4.1 in [13], which is an extension theorem of Lyubich and Phong in [20].

Theorem 6 If $\widetilde{P}$ is the non-negative eigenfunction of corresponding eigenvalue 0 of the system operator such that $\|\widetilde{P}\|=1$, then $\widetilde{P}$ is just the steady-state solution of the system (32). More precisely, the dynamic solution $T(t) \vec{P}(0)$ converges to $\widetilde{P}$ in the norm topology of $X$ as time $t$ approaches infinity, i.e.

$$
\lim _{t \rightarrow \infty}\|T(t) \vec{P}(0)-\widetilde{P}\|=0,
$$

in which $\vec{P}(0)$ is the initial value of the system (32).

On the other hand, we are able to obtain the asymptotic stability of the system by another way. For this purpose, we define the spectral project $E(0, A)$ corresponding to the strictly dominant eigenvalue zero by

$$
E(0, A) \vec{P}=\frac{1}{2 \pi i} \int_{|z|=\varepsilon}(z I-A)^{-1} \vec{P} \mathrm{~d} z=(\vec{P}, \vec{Q}) \widetilde{P}, \quad \forall \vec{P} \in X .
$$

Thus, we can deduce the following theorem from the irreducibility of the semigroup $T(t)$, which 
has been obtained in theorem 4 .

Theorem 7 The semigroup $T(t)$ is Cesàro mean ergodic and the state space $X$ can be decomposed into direct sum of $X=X_{1} \oplus X_{2}$, in which $X_{1}=\operatorname{ker} A=R(E(0, A))$ and $X_{2}=$ $\overline{R(A)}=\operatorname{ker} E(0, A)$. Moreover, for any $\vec{P} \in X$,

$$
\lim _{t \rightarrow \infty}\|T(t) \vec{P}-E(0, A) \vec{P}\|=0 .
$$

Proof By the Definition II 8.1 in [24], we know that the state space $X$ is a $A L$ space. Thus, the statements of the theorem are direct consequences of the lemma 5.1.1 and theorem 5.1.2 in [26].

In particular, if the vector $\vec{P}$ is the initial value $\vec{P}(0)$ of the system (32), then theorem 7 implies theorem 6 since $E(0, A) \vec{P}(0)=(\vec{P}(0), \vec{Q}) \widetilde{P}=\widetilde{P}$. Thus, we obtain the asymptotic stability of the system again. However, another important result of the theorem 6 is the Cesàro mean ergodicity of the semigroup $T(t)$.

\section{Conclusions and further researches}

We obtained the well-posedness and asymptotic stability of the repairable system with three units and vacation by the theory of positive operator semigroups. That is to say the system equations have a unique non-negative dynamic solution and positive steady-state solution and dynamic solution strongly converges to steady-state solution. However, it is pity to enclose the manuscript that we did not give the reliability and exponential stability of the repairable system in this note. We shall show these properties and estimation of the availability of the system in another paper.

\section{Appendix: Proofs of lemmas 1 to 5 and corollaries 1 and 2}

Proof of lemma 1: The identities of the lemma 1 hold since

$$
\begin{gathered}
\int_{0}^{\infty} \mu_{i}(x) \mathrm{e}^{-\int_{0}^{x} \mu_{i}(s) \mathrm{d} s} \mathrm{~d} x=-\int_{0}^{\infty} \mathrm{de}^{-\int_{0}^{x} \mu_{i}(s) \mathrm{d} s}=-\left.\mathrm{e}^{-\int_{0}^{x} \mu_{i}(s) \mathrm{d} s}\right|_{0} ^{\infty}=1, \\
\int_{0}^{\infty} \eta(x) \mathrm{e}^{-\int_{0}^{x} \eta(s) \mathrm{d} s} \mathrm{~d} x=-\int_{0}^{\infty} \mathrm{de}^{-\int_{0}^{x} \eta(s) \mathrm{d} s}=-\left.\mathrm{e}^{-\int_{0}^{x} \eta(s) \mathrm{d} s}\right|_{0} ^{\infty}=1 .
\end{gathered}
$$

Proof of lemma 2: By simple computation we know

$$
b_{1}=1-\int_{0}^{\infty} \mu_{2}(x) \mathrm{e}^{-\int_{0}^{x} \lambda_{3} \mu_{2}(s) \mathrm{d} s} \mathrm{~d} x=1-b_{2} .
$$

Thus, $b_{1}+b_{2}=1$ holds. It is easy to see that

$$
\begin{aligned}
a_{2}+a_{5} & =\lambda_{1} \int_{0}^{\infty} \eta(x) \mathrm{e}^{-\int_{0}^{x} \eta(s) \mathrm{d} s} \int_{0}^{x} \mathrm{e}^{-\left(\lambda_{1}+\lambda_{3}\right) s} \mathrm{~d} s \mathrm{~d} x, \\
a_{3} & =\int_{0}^{\infty} \eta(x) \mathrm{e}^{-\int_{0}^{x} \eta(s) \mathrm{d} s}\left(\mathrm{e}^{-\left(\lambda_{1}+\lambda_{3}\right) x}-\mathrm{e}^{-\left(\lambda_{1}+\lambda_{2}+\lambda_{3}\right) x}\right) \mathrm{d} x, \\
a_{4} & =\int_{0}^{\infty} \eta(x) \mathrm{e}^{-\int_{0}^{x} \eta(s) \mathrm{d} s}\left(\mathrm{e}^{-\left(\lambda_{1}+\lambda_{2}\right) x}-\mathrm{e}^{-\left(\lambda_{1}+\lambda_{2}+\lambda_{3}\right) x}\right) \mathrm{d} x .
\end{aligned}
$$

With the help of lemma 1 we have

$$
\begin{aligned}
& a_{2}+a_{5}+a_{6}+a_{7}+a_{8} \\
= & \left(\lambda_{1}+\lambda_{2}\right) \int_{0}^{\infty} \eta(x) \mathrm{e}^{-\int_{0}^{x} \eta(s) \mathrm{d} s} \int_{0}^{x} \mathrm{e}^{-\left(\lambda_{1}+\lambda_{2}\right) s} \mathrm{~d} s \mathrm{~d} x
\end{aligned}
$$




$$
\begin{aligned}
& +\left(\lambda_{1}+\lambda_{3}\right) \int_{0}^{\infty} \eta(x) \mathrm{e}^{-\int_{0}^{x} \eta(s) \mathrm{d} s} \int_{0}^{x} \mathrm{e}^{-\left(\lambda_{1}+\lambda_{3}\right) s} \mathrm{~d} s \mathrm{~d} x \\
& -\left(\lambda_{1}+\lambda_{2}+\lambda_{3}\right) \int_{0}^{\infty} \eta(x) \mathrm{e}^{-\int_{0}^{x} \eta(s) \mathrm{d} s} \int_{0}^{x} \mathrm{e}^{-\left(\lambda_{1}+\lambda_{2}+\lambda_{3}\right) s} \mathrm{~d} s \mathrm{~d} x \\
= & 1-\int_{0}^{\infty} \eta(x) \mathrm{e}^{-\int_{0}^{x} \eta(s) \mathrm{d} s} \mathrm{e}^{-\left(\lambda_{1}+\lambda_{2}\right) x} \mathrm{~d} x \\
& -\int_{0}^{\infty} \eta(x) \mathrm{e}^{-\int_{0}^{x} \eta(s) \mathrm{d} s} \mathrm{e}^{-\left(\lambda_{1}+\lambda_{3}\right) x} \mathrm{~d} x \\
& +\int_{0}^{\infty} \eta(x) \mathrm{e}^{-\int_{0}^{x} \eta(s) \mathrm{d} s} \mathrm{e}^{-\left(\lambda_{1}+\lambda_{2}+\lambda_{3}\right) x} \mathrm{~d} x .
\end{aligned}
$$

Adding $a_{1}, a_{3}$ and $a_{4}$ to $a_{2}+a_{5}+a_{6}+a_{7}+a_{8}$, we would obtain $\sum_{i=1}^{8} a_{i}=1$.

Proof of lemma 3: When Rez $>0$, it is easy to obtain

$$
\left|\int_{0}^{\infty} \eta(x) \mathrm{e}^{-\int_{0}^{x} z+\eta(s) \mathrm{d} s} \mathrm{~d} x\right|<\int_{0}^{\infty} \eta(x) \mathrm{e}^{-\int_{0}^{x} \eta(s) \mathrm{d} s} \mathrm{~d} x=1 .
$$

When $z=a i$, we have

$$
\left|\int_{0}^{\infty} \eta(x) \mathrm{e}^{-\int_{0}^{x} a i+\eta(s) \mathrm{d} s} \mathrm{~d} x\right| \leq \int_{0}^{\infty} \eta(x) \mathrm{e}^{-\int_{0}^{x} \eta(s) \mathrm{d} s} \mathrm{~d} x=1,
$$

If the equality hold, that is

$$
\left|\int_{0}^{\infty} \eta(x) \mathrm{e}^{-\int_{0}^{x} a i+\eta(s) \mathrm{d} s} \mathrm{~d} x\right|=1,
$$

then

$$
\left|\int_{0}^{\infty} \eta(x) \mathrm{e}^{-\int_{0}^{x} a i+\eta(s) \mathrm{d} s} \mathrm{~d} x\right|^{2}=1 .
$$

It follows from the formula of the module of complex number that

$$
\begin{aligned}
& \left|\int_{0}^{\infty} \eta(x) \mathrm{e}^{-\int_{0}^{x} a i+\eta(s) \mathrm{d} s} \mathrm{~d} x\right|^{2} \\
= & \left(\int_{0}^{\infty} \eta(x) \mathrm{e}^{-\int_{0}^{x} \eta(s) \mathrm{d} s} \cos a x \mathrm{~d} x\right)^{2}+\left(\int_{0}^{\infty} \eta(x) \mathrm{e}^{-\int_{0}^{x} \eta(s) \mathrm{d} s} \sin a x \mathrm{~d} x\right)^{2} \\
= & \int_{0}^{\infty} \eta(x) \mathrm{e}^{-\int_{0}^{x} \eta(s) \mathrm{d} s} \cos a x \mathrm{~d} x \int_{0}^{\infty} \eta(u) \mathrm{e}^{-\int_{0}^{u} \eta(s) \mathrm{d} s} \cos a u \mathrm{~d} u \\
& \quad+\int_{0}^{\infty} \eta(x) \mathrm{e}^{-\int_{0}^{x} \eta(s) \mathrm{d} s} \sin a x \mathrm{~d} x \int_{0}^{\infty} \eta(u) \mathrm{e}^{-\int_{0}^{u} \eta(s) \mathrm{d} s} \sin a u \mathrm{~d} u \\
= & \int_{0}^{\infty} \int_{0}^{\infty} \eta(x) \mathrm{e}^{-\int_{0}^{x} \eta(s) \mathrm{d} s} \eta(u) \mathrm{e}^{-\int_{0}^{u} \eta(s) \mathrm{d} s} \cos a(x-u) \mathrm{d} x \mathrm{~d} u=1 .
\end{aligned}
$$

However, lemma 1 tells us

$$
\int_{0}^{\infty} \int_{0}^{\infty} \eta(x) \mathrm{e}^{-\int_{0}^{x} \eta(s) \mathrm{d} s} \eta(u) \mathrm{e}^{-\int_{0}^{u} \eta(s) \mathrm{d} s} \mathrm{~d} x \mathrm{~d} u=1 .
$$

Thus

$$
\int_{0}^{\infty} \int_{0}^{\infty} \eta(x) \mathrm{e}^{-\int_{0}^{x} \eta(s) \mathrm{d} s} \eta(u) \mathrm{e}^{-\int_{0}^{u} \eta(s) \mathrm{d} s}[1-\cos a(x-u)] \mathrm{d} x \mathrm{~d} u=0 .
$$

Since $\eta(x) \mathrm{e}^{-\int_{0}^{x} \eta(s) \mathrm{d} s} \eta(u) \mathrm{e}^{-\int_{0}^{u} \eta(s) \mathrm{d} s}$ is nonnegative function, the above identity holds if and only if $\cos a(x-u) \equiv 1$, which is equivalent to $a=0$ because $x-u$ is an arbitrary real number. 
Therefore, if $a \neq 0$, then

$$
\left|\int_{0}^{\infty} \eta(x) \mathrm{e}^{-\int_{0}^{x} i a+\eta(s) \mathrm{d} s} \mathrm{~d} x\right| \neq 1 .
$$

This means that the first inequality holds for $z=a i$ and $a \neq 0$. The second inequality will be similarly proven.

Proof of corollary 1: If $z \in \mathbb{C}, \operatorname{Re} z>0$ or $z=a i, a \neq 0$, since $\lambda_{i}$ are positive, it follows that $\left|\lambda_{1}+\lambda_{2}+\lambda_{3}\right|<\left|z+\lambda_{1}+\lambda_{2}+\lambda_{3}\right|$ and $\left|g_{k}\right|<1(k=7,8,9,12)$ hold. Moreover, we have

$$
\sum_{i=0}^{6}\left|g_{i}\right|<\sum_{i=1}^{8} a_{i}=1, \quad\left|g_{10}\right|+\left|g_{11}\right|<b_{1}+b_{2}=1
$$

by lemma 3 and the notations and results of lemma 2. Thus, the statement of this corollary is true by the definition of the diagonally dominant matrix about column(see P. 184 of [21]).

Proof of corollary 2: The function $g_{i}$ given by corollary 2 is analytic in the right half-plane $\{z \in C \mid \operatorname{Re} z>-c\}$. Moreover, we have

$$
\begin{aligned}
& f(z)=|T| \\
= & \left(z+\lambda_{1}+\lambda_{2}+\lambda_{3}\right)\left[1-g_{12}\left(g_{3}+g_{6} g_{8}+g_{5} g_{7}\right)-g_{1} g_{7}-\left(g_{2}+g_{9} g_{4}\right)\right. \\
& \left.\left(g_{11}+g_{8} g_{10} g_{12}\right)\right]-g_{0}\left[\lambda_{2}\left(g_{11}+g_{8} g_{10} g_{12}\right)+\left(\lambda_{3} g_{12}+\lambda_{1} g_{7}\right)\right] .
\end{aligned}
$$

$f(z)$ is then an analytic function in $\{z \in C \mid \operatorname{Re} z>-c\}$. Thus, the conclusion of this corollary results from $\lim _{|\operatorname{Im} z| \rightarrow+\infty}|f(z)|=+\infty$.

Proof of lemma 4: The absolute continuity of the functions $f_{k}(k=1,2, \cdots, 43)$ results from their special expressions. Concerning the absolute integrability, we just give an example. The other function is absolutely integrable is similarly derived. Indeed, we are able to obtain the following estimate

$$
\begin{aligned}
& \int_{0}^{\infty}\left|f_{29}(x)\right| \mathrm{d} x \\
= & \int_{0}^{\infty}\left|\int_{0}^{y} \mathrm{e}^{-\left(\left(1-d_{1}\right) \lambda_{1}+\lambda_{3}\right)(y-\tau)} \mathrm{e}^{-\int_{\tau}^{x} z+\mu_{2}(s) \mathrm{d} s} \int_{0}^{\infty} \mu_{1}(u) \int_{0}^{u} \mathrm{e}^{-\int_{r}^{u} z+\mu_{1}(s) \mathrm{d} s} q_{4}(r, \tau) \mathrm{d} r \mathrm{~d} u \mathrm{~d} \tau \mathrm{d} y\right| \mathrm{d} x \\
\leq & \int_{0}^{\infty} \int_{0}^{x} \int_{0}^{y}\left|\mathrm{e}^{-\int_{\tau}^{x} z+\mu_{2}(s) \mathrm{d} s} \mathrm{e}^{-\left(\left(1-d_{1}\right) \lambda_{1}+\lambda_{3}\right)(y-\tau)} \int_{0}^{\infty} \mu_{1}(u) \int_{0}^{u} \mathrm{e}^{-\int_{r}^{u} z+\mu_{1}(s) \mathrm{d} s} q_{4}(r, \tau) \mathrm{d} r \mathrm{~d} u\right| \mathrm{d} \tau \mathrm{d} y \mathrm{~d} x \\
\leq & \frac{1}{c_{2}} \int_{0}^{\infty} \int_{0}^{x} \int_{0}^{y} \mathrm{e}^{c_{2} \tau} \mathrm{e}^{-\left(\left(\left|1-d_{1}\right|\right) \lambda_{1}+\lambda_{3}\right)(y-\tau)} \int_{0}^{\infty} \int_{0}^{u} \mathrm{e}_{0}^{\int_{0}^{r} \mu_{1}(s) \mathrm{d} s}\left|q_{4}(r, \tau)\right| \mathrm{d} r \mathrm{de}^{-\int_{0}^{u} \mu_{1}(s) \mathrm{d} s} \mathrm{~d} \tau \mathrm{d} y \mathrm{de} \mathrm{e}^{-c_{2} x} \\
= & \frac{1}{c_{2}} \int_{0}^{\infty} \int_{0}^{x} \mathrm{e}^{-\left(\left(\left|1-d_{1}\right|\right) \lambda_{1}+\lambda_{3}+c_{2}\right)(x-\tau)} \int_{0}^{\infty}\left|q_{4}(u, \tau)\right| \mathrm{d} u \mathrm{~d} \tau \mathrm{d} x \\
= & -\frac{1}{c_{2}} \frac{1}{\left|\left(1-d_{1}\right)\right| \lambda_{1}+\lambda_{3}+c_{2}} \int_{0}^{\infty} \int_{0}^{x} \mathrm{e}^{\left(\left(1-d_{1}\right) \lambda_{1}+\lambda_{3}+c_{2}\right) \tau} \int_{0}^{\infty}\left|q_{4}(u, \tau)\right| \mathrm{d} u \mathrm{~d} \tau \mathrm{de}^{-\left(\left(1-d_{1}\right) \lambda_{1}+\lambda_{3}+c_{2}\right) x} \\
\leq & \frac{1}{c_{2}} \frac{1}{\lambda_{3}+c_{2}} \int_{0}^{\infty} \int_{0}^{\infty}\left|q_{4}(u, x)\right| \mathrm{d} u \mathrm{~d} x<\infty,
\end{aligned}
$$

by the general hypotheses and integration by parts. Thus, $f_{29}(x)$ is an absolutely integrable function.

Proof of lemma 5: $e_{l}(l=0,1, \cdots, 7) \in \mathbb{C}$ is equivalent to the module $\left|e_{l}\right|$ of $e_{l}$ is finite. 
Only one example is given here. For instance, the module $\left|e_{0}\right|$ is finite due to

$$
\begin{aligned}
\left|e_{0}\right| & =\left|\int_{0}^{\infty} \eta(x) f_{7}(x) \mathrm{d} x\right| \\
& \leq \int_{0}^{\infty} \eta(x) \int_{0}^{x}\left|\mathrm{e}^{-\int_{\tau}^{x} z+\lambda_{1}+\lambda_{2}+\lambda_{3}+\mu_{3}(s) \mathrm{d} s} q_{7}(\tau) \mathrm{d} \tau\right| \mathrm{d} x \\
& \leq-\int_{0}^{\infty} \int_{0}^{x}\left|\mathrm{e}^{\int_{0}^{\tau} \eta(s) \mathrm{d} s} q_{7}(\tau) \mathrm{d} \tau\right| \mathrm{de}^{-\int_{0}^{x} \eta(s) \mathrm{d} s} \\
& =\int_{0}^{\infty}\left|q_{7}(x)\right| \mathrm{d} x<\infty
\end{aligned}
$$

in which the same techniques of the above lemma are used.

\section{Acknowledgments}

The authors would like to thank the referees for their helpful suggestions and comments.

This paper is supported by the National Natural Science Foundation of China (Grant No.11201037).

\section{References}

[1] Song S B, Deng Y L. Reliability analysis of a three-unit system in a changing environment. Microelectronics Reliability, 1993, 33: 637-640.

[2] Li W, Alfa A S, Zhao Y Q. Stochastic analysis of a repairable system with three units and two repair facilities. Microelectronics Reliability, 1998, 38: 585-595.

[3] Kovalenko A I. Analysis of the reliability of a three-component system with renewal. Journal of Mathematical Sciences, 2001, 103: 273-277.

[4] Hu L M, Yue D Q, Tian R L. Reliability analysis of a three-unit system with $n$ failure modes and priority. Journal of Computer Information Systems, 2009, 5: 253-258.

[5] Guo L N, Xu H, Gao C, et al. Stability analysis of a new kind $N$-units series repairable system. Applied Mathematical Modelling, 2011, 35(1): 202-217.

[6] Su B H, Shi D H. Reliability analysis of $n$-unit series systems with multiple vacations of a repairman. Mathematical Statistics and Applied Probability, 1995, 10: 78-82.

[7] $\mathrm{Hu} \mathrm{L} \mathrm{M,} \mathrm{Tian} \mathrm{R} \mathrm{L,} \mathrm{Wu} \mathrm{J} \mathrm{B,} \mathrm{et} \mathrm{al.} \mathrm{Reliability} \mathrm{analysis} \mathrm{of} \mathrm{serie-parallel} \mathrm{repairable} \mathrm{system} \mathrm{consisting} \mathrm{of}$ three-unit with vacation. Journal of Yanshan University, 2007, 31: 299-303.

[8] $\mathrm{Hu}$ L M, Li J D. Reliability analysis of a three-unit system with vacation and priority. ICIC Express Letters, 2009, 3: 171-176.

[9] Hu L M, Yue D Q, Li J D. Probabilistic analysis of a series-parallel repairable system with three units and vacation. Applied Mathematical Modelling, 2010, 34: 2711-2721.

[10] Gupur G, Li X Z. Semigroup method for a mathematical model in reliability analysis. Journal of Systems Science and Systems Engineering, 2001, 10: 137-147.

[11] Hu W W, Shen Z F, Xin Y H, et al. Exponential stability of a repairable system with imperfect switching mechanism. Asymptotic Analysis, 2007, 54: 93-102.

[12] Shen Z F, Hu X X, Fan W F, et al. Exponential asymptotic property of a parallel repairable system with warm standby under common-cause failure. Journal of Mathematical Analysis and Applications, 2008, 341: $457-466$.

[13] Wang W L, Xu G Q. Stability analysis of a complex standby system with constant waiting and different repairman criteria incorporating environmental failure. Applied Mathematical Modelling, 2009, 33: 724743.

[14] Zheng F, Zhu G T, Gao C. Well-posedness and stability of the repairable system with $N$ failure modes and one standby unit. Journal of Mathematical Analysis and Applications, 2011, 375: 174-184.

[15] Zheng F, Zhu G T. The Exponential stability of a repairable system with checking time. Advance in Mathematics, 2010, 39(5): 568-576. 
[16] Ma L X, Xu G Q. Analysis of a deteriorating cold standby system with priority. WSEAS Transactions on Mathematics, 2011, 10(2): 84-94.

[17] Yuan W Z, Xu G Q. Modelling of a deteriorating system with repair satisfying general distribution. Applied Mathematics and Computation, 2012, 218(11): 6340-6350.

[18] Wang H X, Xu G Q. A cold system with two different components and a single vacation of the repairman. Applied Mathematics and Computation, 2012, 219(5): 2634-2657.

[19] Guo L N, Xu H B, Gao C, et al. Stability analysis of a new kind series system. IMA Journal of Applied Mathematics, 2010, 75: 439-460.

[20] Lyubich Y I, Phong V Q. Asymptotic stability of linear differential equations in Banach spaces. Studia Mathematica, 1988, 88: 37-42.

[21] Meyer C D. Matrix analysis and applied linear algebra. Cambridge University Press, 2000.

[22] Gupur G, Li X Z, Zhu G T. Functional analysis method in queueing theory. Hertfordshire, Research Information Ltd: United Kingdom, 2001.

[23] Nager R. One-parameter semigroup of positive operators. New York: Springer-Verlag, 1986.

[24] Schaefer H H. Babach lattice and positive operator. Springer-Verlag, 1974.

[25] Engel K J, Nagel R. A short course on operator semigroups. Springer, 2006.

[26] Radle R. Semigroups applied to transport and queueing processes. Doctoral Dissertatian of Tubingen University, 2006. 